\title{
Stochastic Models to Qualify Stem Tapers
}

\author{
Edmundas Petrauskas ${ }^{1}$, Petras Rupšys ${ }^{1, * \mathbb{C}}$, Martynas Narmontas ${ }^{1}$, Marius Aleinikovas ${ }^{2}$, \\ Lina Beniušienè ${ }^{2}$ and Benas Šilinskas ${ }^{2}$ \\ 1 Agriculture Academy, Vytautas Magnus University, Universiteto g. 20-214, Akademija, 53361 Kaunas, \\ Lithuania; edmundas.petrauskas@vdu.lt (E.P.); martynas.narmontas@vdu.lt (M.N.) \\ 2 Lithuanian Research Centre for Agriculture and Forestry, Institute of Forestry, Liepu str.1, Girionys, \\ 53101 Kaunas distr., Lithuania; marius.aleinikovas@lammc.lt (M.A.); lina.beniusiene@lammc.lt (L.B.); \\ benas.silinskskas@lammc.lt (B.Š.) \\ * Correspondence: petras.rupsys@vdu.lt
}

Received: 9 March 2020; Accepted: 14 April 2020; Published: 15 April 2020

\begin{abstract}
This study examines the performance of 11 tree taper models to predict the diameter of bark at any given height and the total stem volume of eight dominant tree species in the boreal forests of Lithuania. Here, we develop eight new models using stochastic differential equations (SDEs). The symmetrical Vasicek model and asymmetrical Gompertz model are used to describe tree taper evolution, as well as geometric-type diffusion processes. These models are compared with those traditionally used for four tree taper models by using performance statistics and residual analysis. The observed dataset consists of longitudinal measurements of 3703 trees, representing the eight dominant tree species in Lithuania (pine, spruce, oak, ash, birch, black alder, white alder, and aspen). Overall, the best goodness of fit statistics of diameter predictions produced the SDE taper models. All results have been implemented in the Maple computer algebra system using the "Statistics" and "VectorCalculus" packages.
\end{abstract}

Keywords: stem taper; stochastic differential equation; probability density function; maximum likelihood procedure

\section{Introduction}

Stochastic differential equations (SDEs) were developed at the beginning of the 20th century to quantify aspects of stochastic processes. The pioneering work of Bachelier [1] was primarily motivated by problems associated with introducing a mathematical model of Brownian motion and its use for valuing stock options. Although applications of stochastic calculus theory can be highly technical, fundamental concepts of SDE theory are not difficult to understand. Moreover, they are profound in the sense that they apply to situations in which commonly used models produce unsatisfactory results. Fundamental SDE theory is defined on random variables. The universality of random processes accounts for the wide range of applications of the theory, including human population [2], forestry [3,4], biology [5], and epidemiology [6]. In biological systems, SDEs are used in place of deterministic models, obtained by including a noise term in the ordinary differential equation of the respective deterministic model [7]. The main reason to develop SDE models is the capacity to model highly nonlinear biological dynamic [8,9]. The fundamental advantage of stochastic dynamic models over deterministic models is that they combine both the deterministic and stochastic elements of dynamic systems, where the stochasticity is affected by the outside factors such as weather. This makes stochastic modeling a powerful tool in the hands of practitioners in fields for which population growth is a critical determinant of various outcomes. There is a general tendency in the population growth modeling literature to favor flexible techniques that represent features of multivariate data as well as possible $[10,11]$. Therefore, multivariate SDEs describing population growth models contain both the 
main effects and interaction effects involved in models using a variance-covariance matrix, improving the potential to interpret the data more informatively [12] and inferring the causality in a statistical sense as a type of dependence of the multivariate random variables [13].

The mathematical link between tree diameters at any specified height where the height is known is known as a tree (stem) taper equation. The random variables of interest in the present study are the diameter at any specified height and the relative diameter at any specified relative height. Stem taper equations are traditionally used to estimate diameters at any specified tree height, conversely, to estimate a corresponding height at any specified diameter, and to estimate the total tree volume or the volume of specified products if given the specified dimensions. Stem taper equations are the ultimate base of computer algorithms for calculating stem volumes of any assortment of structures. In the forest literature, many papers have already looked into the link between the relative diameter and relative height of trees. However, the most published models are deterministic regression models. It is advisable to divide taper equations into five categories, namely, (1) segmented polynomial taper equations [14], (2) variable exponential equations [15-17], (3) artificial neural network (ANN) equations [18], (4) fundamental mechanisms of stem profile formation equations [19,20], and (5) diffusion process equations [21]. The first four categories include deterministic taper models that describe the variation of the stem form by means of a single equation, where the diameter at any specified stem height is the dependent variable, while the diameter at breast height and overall height are the independent variables. Some researchers demonstrated that including a variable of crown size, stand density, age, and others as a predictor could help explain variation in tree shape [22].

A segmented polynomial taper equation describes the profile of a stem by separating it into two or more segments. The bottom bole section is modeled as a frustum of a neiloidal solid, the middle of the bole is assumed to take the shape of a frustum of a paraboloidal solid, and the top portion is assumed to be conoid [23]. Traditionally, three curves are welded together by incorporating the joint points between the three segments. A variable exponential equation models developed by Kozak [24] are known as the best for stem taper estimation. A variable exponential model is more precise comparing to segmented polynomial model. Both segmented polynomial and variable exponential regression models urge that confirmed some statistical assumptions such as: independence, normality, homoscedasticity, and much more. Artificial neural network and stochastic differential models are capable of handling non-normality and nonlinearity in a stem taper system. Generally, the segmented polynomial, variable exponential, and artificial neural network models are empirical, based on large data sets, and static. Mechanistic stem taper models of stem development are derived from simplified models of mechanisms of tree form development and plant physiology knowledge [25]. From a practical point of view this study focused on the segmented polynomial, variable exponential, and stochastic differential equations models.

The shape of a tree stem is related to the growth environment of a tree, where different types of irregularities, such as wounds, coarse bark areas, and branches vary among trees of the same species [21]. A goodness of fit of a model is related with the number of incorporated independent variables, such as the planting density, fertilization, thinning, and crown height. Unfortunately, insignificant improvement has been observed in model performance when including additional dimensions as covariate variables. Studies from the last few decades have incorporated mixed effect (fixed and random effect) modeling techniques in the development of taper equations [26]. The theoretical investigation of this technique accounts for both the within- and between-tree variation in stem taper forms $[17,27,28]$. From the practical point of view, a mixed effect modeling technique needs additional prior information (measurements) for predicting the diameter at any specified height for a new stem. Random effects can be calibrated and an adjusted response (stem-specific), rather than a mean response (fixed effect), can be recommended if additional stem diameter measurements are available.

Diffusion processes based on stem taper models are not affected by the mathematical form, because we start from the evolution of the probability density function of the relative diameter [21]. Another 
characteristic of newly developed stem taper models is that the derived normal and log-normal probability density functions of the solutions of the SDEs enable us to define the evolution of any central moment (mean, variance, and so on), quartile, $p$-quantile $(0<p<1)$, and coefficient of variation of the diameter at any specified height when both stem diameter and height are given. Such models can reflect a more complex variation in particular stem tapers due to the initial conditions represented by measurement of the tree diameter at the tree height, $h=0$, or its distribution.

Motivated by the stem taper equations [14,16], this work proposes a few new models based on diffusion processes. First, the Gompertz-, Vasicek- and geometric-type diffusions are used in stem taper models to improve the predictions of diameter at any specified height and the total stem volume. Finally, a comparison mechanism based on data from the eight dominant tree species in Lithuania (pine, spruce, oak, ash, birch, black alder, white alder, and aspen) is proposed to further enhance the robustness of newly developed models.

\section{Materials and Methods}

\subsection{SDE Stem Tapers}

This study focuses on univariate continuous diffusion processes $Y^{i}(x), i=1, \ldots, M$, evolving in $M$ different individuals (tree stems). We suppose that the evolution of the relative diameter $Y^{i}=\frac{D}{d^{i}}$ against the relative height $x^{i}=\frac{h}{h^{i}}\left(x^{i} \in[0 ; 1]\right.$, in the sequel $\left.x\right)$ is expressed by the Itô-type stochastic differential equation [29], where $D$ is the diameter at any specified height $h, d^{i}$ is the diameter at breast height of the $\mathrm{i}$-th tree, and $h^{i}$ is the total stem height of the $\mathrm{i}$-th tree. In the following, we will consider three different univariate diffusion models given by the SDEs with explicitly known transition distributions.

The Gompertz-type stochastic differential equation of the relative diameter against the relative height is formulated as follows:

$$
d Y^{i}(x)=\left[\alpha_{1} Y^{i}(x)-\beta_{1} Y^{i}(x) \ln \left(Y^{i}(x)\right)\right] d x+\sigma_{1} Y^{i}(x) d W_{1}^{i}(x), i=1, \ldots, M,
$$

where $\alpha_{1}, \beta_{1}$, and $\sigma_{1}$ are fixed effects parameters (identical for all stems from the specified tree species) and $y_{0}^{i}$ is the initial relative diameter. In this study, we focus on two initial distributions, namely, the deterministic initial value $Y^{i}(0)=y_{0}^{i}$, which is a degenerate initial distribution $P\left(Y^{i}(0)=y_{0}^{i}\right)=1$, and a log-normal distribution, $Y^{i}(0) \sim L N_{1}\left(\mu_{0} ; \sigma_{0}^{2}\right)$. Here, $W_{1}^{i}(x), i=1, \ldots, M$ represents independent standard Brownian motions. The diffusion process, $Y^{i}(x)$, conditioned on the initial value $y_{0}^{i}$, has a $\log$-normal distribution where $L N_{1}\left(\mu_{1}\left(x \mid \alpha_{1}, \beta_{1}, \sigma_{1}, y_{0}^{i}\right) ; v_{1}\left(x \mid \beta_{1}, \sigma_{1}\right)\right)$ with the conditional mean, variance, and density, respectively.

$$
\begin{gathered}
\mu_{1}\left(x \mid \alpha_{1}, \beta_{1}, \sigma_{1}, y_{0}^{i}\right)=e^{-\beta_{1} x} \ln \left(y_{0}^{i}\right)+\left(\frac{1-e^{-\beta_{1} x}}{\beta_{1}}\right)\left(\alpha_{1}-\frac{\sigma_{1}^{2}}{2}\right), \\
v_{1}\left(x \mid \beta_{1}, \sigma_{1}\right)=\frac{\sigma_{1}^{2}}{2 \beta_{1}}\left(1-e^{-2 \beta_{1} x}\right), \\
p_{G}\left(y, x \mid \alpha_{1}, \beta_{1}, \sigma_{1}, y_{0}^{i}\right)=\frac{1}{y \sqrt{2 \pi v_{1}\left(x \mid \beta_{1}, \sigma_{1}\right)}} \exp \left(-\frac{\left(\ln y-\mu_{1}\left(x \mid \alpha_{1}, \beta_{1}, \sigma_{1}, y_{0}^{i}\right)\right)^{2}}{2 v_{1}\left(x \mid \beta_{1}, \sigma_{1}\right)}\right) .
\end{gathered}
$$

A geometric Brownian motion stochastic differential equation of the relative diameter against the relative height is formulated as follows:

$$
d Y^{i}(x)=\alpha_{2} Y^{i}(x) d x+\sigma_{2} Y^{i}(x) d W_{2}^{i}(x), i=1, \ldots, M,
$$


where $\alpha_{2}$, and $\sigma_{2}$ are fixed effect parameters (identical for all stems from the specified tree species) and $W_{2}^{i}(x)$ denotes independent standard Brownian motions. This process, $Y^{i}(x)$, conditioned on the initial value $y_{a_{*}}^{i}$, thus is $P\left(Y^{i}\left(a_{*}\right)=y_{a_{*}}^{i}\right)=1, a_{*} \in\left\{a_{0}, a_{1}\right\}$ and has a log-normal distribution where $L N_{1}\left(\mu_{2}\left(x \mid \alpha_{2}, \sigma_{2}, y_{a_{*}}^{i}, a_{*}\right) ; v_{2}\left(x \mid \sigma_{2}, a_{*}\right)\right)$ with the conditional mean, variance, and density defined, respectively, as follows:

$$
\begin{gathered}
\mu_{2}\left(x \mid \alpha_{2}, \sigma_{2}, y_{a_{*}}^{i}, a_{*}\right)=\ln \left(y_{a_{*}}^{i}\right)+\left(\alpha_{2}-\frac{\sigma_{2}^{2}}{2}\right)\left|x-a_{*}\right|, \\
v_{2}\left(x \mid \sigma_{2}, a_{*}\right)=\sigma_{2}^{2}\left|x-a_{*}\right|, \\
p_{g}\left(y, x \mid \alpha_{2}, \sigma_{2}, y_{a_{*}}^{i}, a_{*}\right)=\frac{1}{y \sqrt{2 \pi v_{2}\left(x \mid \sigma_{2}, a_{*}\right)}} \exp \left(-\frac{\left(\ln y-\mu_{2}\left(x \mid \alpha_{2}, \sigma_{2}, y_{a_{*}}^{i} a_{*}\right)\right)^{2}}{2 v_{2}\left(x \mid \sigma_{2}, a_{*}\right)}\right) .
\end{gathered}
$$

The Vasicek-type stochastic differential equation of the relative diameter against the relative height is formulated as follows:

$$
d Y^{i}(x)=\beta_{3}\left(\alpha_{3}-Y^{i}(x)\right) d x+\sigma_{3} d W_{3}^{i}(x), i=1, \ldots, M,
$$

where $\alpha_{3}, \beta_{3}$, and $\sigma_{3}$ are fixed effects parameters (identical for all stems from the specified tree species) and $W_{3}^{i}(x)$ denotes independent standard Brownian motions. This process, $Y^{i}(x)$, conditioned on the initial value $y_{a_{* *}}^{i}$, thus is $P\left(Y^{i}\left(a_{* *}\right)=y_{a_{* *}}^{i}\right)=1, a_{* *} \in\left\{a_{0}, a_{1}, 1\right\}$ and has a normal distribution $N_{1}\left(\mu_{3}\left(x \mid \alpha_{3}, \beta_{3}, y_{a_{* *}}^{i}, a_{* *}\right) ; v_{3}\left(x \mid \beta_{3}, \sigma_{3}, a_{* *}\right)\right)$ with the conditional mean, variance, and density, respectively.

$$
\begin{gathered}
\mu_{3}\left(x \mid \alpha_{3}, \beta_{3}, y_{a_{* *}}^{i}, a_{* *}\right)=\alpha_{3}-\left(y_{a_{* *}}^{i}-\alpha_{3}\right) \cdot e^{-\beta_{3}\left|x-a_{* *}\right|}, \\
v_{3}\left(x \mid \beta_{3}, \sigma_{3}, a_{* *}\right)=\frac{\sigma_{3}^{2}}{2 \beta_{3}}\left(1-e^{-2 \beta_{3}\left|x-a_{* *}\right|}\right), \\
p_{V}\left(y, x \mid \alpha_{3}, \beta_{3}, \sigma_{3}, y_{a_{* *}}^{i}, a_{* *}\right)=\frac{1}{\sqrt{2 \pi v_{3}\left(x \mid \beta_{3}, \sigma_{3}, a_{* *}\right)}} \exp \left(-\frac{\left(y-\mu_{3}\left(x \mid \alpha_{3}, \beta_{3}, y_{a_{* *}}^{i}, a_{* *}\right)\right)^{2}}{2 v_{3}\left(x \mid \beta_{3}, \sigma_{3}, a_{* *}\right)}\right) .
\end{gathered}
$$

In this study, newly developed stem tapers are defined (as segmented models) by using one or two joining points ( $a_{0}$ and $a_{1}$ ) to weld three stochastic processes, defined by Equations (1), (5), and (9). In the sequel, the fixed effects parameters for the bottom part of a stem are listed by index $B$, the middle part by index $M$, and for the top part, they are listed by index $T$. The stem taper SDE models with two joining points $a_{0}$ and $a_{1}$ are defined in the following forms, respectively:

$$
\text { Model } 1 d Y^{i}(x)=\left\{\begin{array}{l}
{\left[\alpha_{B} Y^{i}(x)-\beta_{B} Y^{i}(x) \ln \left(Y^{i}(x)\right)\right] d x+\sigma_{B} Y^{i}(x) d W_{B}^{i}(x), P\left(Y^{i}\left(x_{0}\right)=y_{0}^{i}\right)=1, x<a_{0}} \\
\alpha_{M} Y^{i}(x) d x+\sigma_{M} Y^{i}(x) d W_{M}^{i}(x), P\left(Y^{i}\left(a_{0}\right)=1\right)=1, a_{0} \leq x<a_{1} \\
\beta_{T}\left(\alpha_{T}-Y^{i}(x)\right) d x+\sigma_{T} d W_{T}^{i}(x), P\left(Y^{i}(1)=0\right)=1, a_{1} \leq x \leq 1
\end{array},\right.
$$

$$
\begin{gathered}
\text { Model } 2 d Y^{i}(x)=\left\{\begin{array}{l}
{\left[\alpha_{B} Y^{i}(x)-\beta_{B} Y^{i}(x) \ln \left(Y^{i}(x)\right)\right] d x+\sigma_{B} Y^{i}(x) d W_{B}^{i}(x), P\left(Y^{i}\left(x_{0}\right)=y_{0}^{i}\right)=1, x \leq a_{0}} \\
\alpha_{M} Y^{i}(x) d x+\sigma_{M} Y^{i}(x) d W_{M}^{i}(x), P\left(Y^{i}\left(a_{1}\right)=y_{a_{1}}^{i}\right)=1, a_{0}<x \leq a_{1} \\
\beta_{T}\left(\alpha_{T}-Y^{i}(x)\right) d x+\sigma_{T} d W_{T}^{i}(x), P\left(Y^{i}(1)=0\right)=1, a_{1}<x \leq 1
\end{array}\right. \\
\text { Model 3d } Y^{i}(x)=\left\{\begin{array}{l}
{\left[\alpha_{B} Y^{i}(x)-\beta_{B} Y^{i}(x) \ln \left(Y^{i}(x)\right)\right] d x+\sigma_{B} Y^{i}(x) d W_{B}^{i}(x), P\left(Y^{i}\left(x_{0}\right)=\gamma\right)=1, x<a_{0}} \\
\alpha_{M} Y^{i}(x) d x+\sigma_{M} Y^{i}(x) d W_{M}^{i}(x), P\left(Y^{i}\left(a_{0}\right)=1\right)=1, a_{0} \leq x<a_{1} \\
\beta_{T}\left(\alpha_{T}-Y^{i}(x)\right) d x+\sigma_{T} d W_{T}^{i}(x), P\left(Y^{i}(1)=0\right)=1, a_{1} \leq x \leq 1
\end{array}\right.
\end{gathered}
$$


where $\gamma$ is a random variable, log-normally distributed, where $\gamma \sim L_{1}\left(\mu_{0} ; \sigma_{0}^{2}\right)$ with the unknown fixed effect parameters $\mu_{0}$ and $\sigma_{0}^{2}$ to be estimated. On the other side, the stem butt of the $\mathrm{i}$-th tree is assumed to be free.

Model $4 d Y^{i}(x)=\left\{\begin{array}{l}{\left[\alpha_{B} Y^{i}(x)-\beta_{B} Y^{i}(x) \ln \left(Y^{i}(x)\right)\right] d x+\sigma_{B} Y^{i}(x) d W_{B}^{i}(x), P\left(Y^{i}\left(x_{0}\right)=y_{0}^{i}\right)=1, x<a_{0}} \\ \beta_{M}\left(\alpha_{M}-Y^{i}(x)\right) d x+\sigma_{M} d W_{M}^{i}(x), P\left(Y^{i}\left(a_{0}\right)=1\right)=1, a_{0} \leq x<a_{1} \\ \beta_{T}\left(\alpha_{T}-Y^{i}(x)\right) d x+\sigma_{T} d W_{T}^{i}(x), P\left(Y^{i}(1)=0\right)=1, a_{1} \leq x \leq 1\end{array}\right.$,

Model $5 d Y^{i}(x)=\left\{\begin{array}{l}{\left[\alpha_{B} Y^{i}(x)-\beta_{B} Y^{i}(x) \ln \left(Y^{i}(x)\right)\right] d x+\sigma_{B} Y^{i}(x) d W_{B}^{i}(x), P\left(Y^{i}\left(x_{0}\right)=y_{0}^{i}\right)=1, x \leq a_{0}} \\ \beta_{M}\left(\alpha_{M}-Y^{i}(x)\right) d x+\sigma_{M} d W_{M}^{i}(x), P\left(Y^{i}\left(a_{1}\right)=y_{a_{1}}^{i}\right)=1, a_{0}<x \leq a_{1} \\ \beta_{T}\left(\alpha_{T}-Y^{i}(x)\right) d x+\sigma_{T} d W_{T}^{i}(x), P\left(Y^{i}(1)=0\right)=1, a_{1}<x \leq 1\end{array}\right.$

Model $6 d Y^{i}(x)=\left\{\begin{array}{l}{\left[\alpha_{B} Y^{i}(x)-\beta_{B} Y^{i}(x) \ln \left(Y^{i}(x)\right)\right] d x+\sigma_{B} Y^{i}(x) d W_{B}^{i}(x), P\left(Y^{i}\left(x_{0}\right)=\gamma\right)=1, x<a_{0}} \\ \beta_{M}\left(\alpha_{M}-Y^{i}(x)\right) d x+\sigma_{M} d W_{M}^{i}(x), P\left(Y^{i}\left(a_{0}\right)=1\right)=1, a_{0} \leq x<a_{1} \\ \beta_{T}\left(\alpha_{T}-Y^{i}(x)\right) d x+\sigma_{T} d W_{T}^{i}(x), P\left(Y^{i}(1)=0\right)=1, a_{1} \leq x \leq 1\end{array}\right.$,

where $\gamma$ is a random variable, log-normally distributed, where $\gamma \sim L N_{1}\left(\mu_{0} ; \sigma_{0}^{2}\right)$ with the unknown fixed effect parameters $\mu_{0}$ and $\sigma_{0}^{2}$ is to be estimated.

The stem taper SDE models with one joining point $a_{0}$ are defined in the following forms (fixed effects parameters for the bottom part of a stem are listed by index $B$, and, for the top part, these are listed by index T):

Model $7 d Y^{i}(x)=\left\{\begin{array}{l}{\left[\alpha_{B} Y^{i}(x)-\beta_{B} Y^{i}(x) \ln \left(Y^{i}(x)\right)\right] d x+\sigma_{B} Y^{i}(x) d W_{B}^{i}(x), P\left(Y^{i}\left(x_{0}\right)=y_{0}^{i}\right)=1, x \leq a_{0}} \\ \beta_{T}\left(\alpha_{T}-Y^{i}(x)\right) d x+\sigma_{T} d W_{T}^{i}(x), P\left(Y^{i}(1)=0\right)=1, a_{0}<x \leq 1\end{array}\right.$,

Model $8 d Y^{i}(x)=\left\{\begin{array}{l}{\left[\alpha_{B} Y^{i}(x)-\beta_{B} Y^{i}(x) \ln \left(Y^{i}(x)\right)\right] d x+\sigma_{B} Y^{i}(x) d W_{B}^{i}(x), P\left(Y^{i}\left(x_{0}\right)=\gamma\right)=1, x \leq a_{0}} \\ \beta_{T}\left(\alpha_{T}-Y^{i}(x)\right) d x+\sigma_{T} d W_{T}^{i}(x), P\left(Y^{i}(1)=0\right)=1, a_{0}<x \leq 1\end{array}\right.$,

where $\gamma$ is a random variable, log-normally distributed, where $\gamma \sim L N_{1}\left(\mu_{0} ; \sigma_{0}^{2}\right)$ with the unknown fixed effect parameters $\mu_{0}$ and $\sigma_{0}^{2}$ is to be estimated.

\subsection{Mean Trends of Stem Tapers}

Using the conditional probability density functions, defined by Equations (2)-(4), (6)-(8), and (10)-(12), we can define the mean trajectories, $m_{k}(\cdot)(k=1, \ldots, 8)$, for Models $1-8$ in the following forms (the estimates of the fixed effect parameters are denoted by adding 'hat'), respectively:

$$
\begin{gathered}
\text { Model } 1 m_{1}\left(x, d^{i}, h^{i}\right)=\left\{\begin{array}{l}
d^{i} \exp \left(\mu_{1}\left(\frac{x}{h^{i}} \mid \hat{\alpha}_{B}, \hat{\beta}_{B}, \hat{\sigma}_{B}, \frac{d_{0}^{i}}{d^{i}}\right)+\frac{1}{2} v_{1}\left(\frac{x}{h^{i}} \mid \hat{\beta}_{B}, \hat{\sigma}_{B}\right)\right), \frac{x}{h^{i}}<\frac{1.3}{h^{i}} \\
d^{i} \exp \left(\mu_{2}\left(\frac{x}{h^{i}} \mid \hat{\alpha}_{M}, \hat{\sigma}_{M}, 1 .\right)+\frac{1}{2} v_{2}\left(\frac{x}{h^{i}} \mid \hat{\sigma}_{M}, \frac{1.3}{h^{i}}\right)\right), \frac{1.3}{h^{i}} \leq \frac{x}{h^{i}}<a_{1}, \\
d^{i} \mu_{3}\left(\frac{x}{h^{i}} \mid \hat{\alpha}_{T}, \hat{\beta}_{T}, 0\right), a_{1} \leq \frac{x}{h^{i}} \leq 1
\end{array}\right. \\
\text { Model } 2 m_{2}\left(x, d^{i}, h^{i}\right)=\left\{\begin{array}{l}
d^{i} \exp \left(\mu_{1}\left(\frac{x}{h^{i}} \mid \hat{\alpha}_{B}, \hat{\beta}_{B}, \hat{\sigma}_{B}, \frac{d_{0}^{i}}{d^{i}}\right)+\frac{1}{2} v_{1}\left(\frac{x}{h^{i}} \mid \hat{\beta}_{B}, \hat{\sigma}_{B}\right)\right), \frac{x}{h^{i}} \leq a_{0} \\
d^{i} \exp \left(\mu_{2}\left(\frac{x}{h^{i}} \mid \hat{\alpha}_{M}, \hat{\sigma}_{M}, \mu_{3}\left(a_{1} \mid \hat{\alpha}_{T}, \hat{\beta}_{T}, 0\right)\right)+\frac{1}{2} v_{2}\left(\frac{x}{h^{i}} \mid \hat{\sigma}_{M}, a_{1}\right)\right), a_{0}<\frac{x}{h^{i}} \leq a_{1} \\
d^{i} \mu_{3}\left(\frac{x}{h^{i}} \mid \hat{\alpha}_{T}, \hat{\beta}_{T}, 0\right), a_{1}<\frac{x}{h^{i}} \leq 1
\end{array}\right.
\end{gathered}
$$




$$
\begin{aligned}
& \text { Model } 3 m_{3}\left(x, d^{i}, h^{i}\right)=\left\{\begin{array}{l}
d^{i} \cdot \exp \left(\mu_{0}\left(\mu_{1}\left(\frac{x}{h^{i}} \mid \hat{\alpha}_{B}, \hat{\beta}_{B}, \hat{\sigma}_{B}, \hat{\gamma}\right)+\frac{1}{2} v_{1}\left(\frac{x}{h^{i}} \mid \hat{\beta}_{B}, \hat{\sigma}_{B}\right)\right)\right), \frac{x}{h^{i}}<a_{0} \\
d^{i} \cdot \exp \left(\mu_{2}\left(\frac{x}{h^{i}} \mid \hat{\alpha}_{M}, \hat{\sigma}_{M}, \mu_{3}\left(a_{1} \mid \hat{\alpha}_{T}, \hat{\beta}_{T}, 0\right)\right)+\frac{1}{2} v_{2}\left(\frac{x}{h^{i}} \mid \hat{\sigma}_{M}, a_{1}\right)\right), a_{0} \leq \frac{x}{h^{i}}<a_{1} \\
d^{i} \cdot \mu_{3}\left(\frac{x}{h^{i}} \mid \hat{\alpha}_{T}, \hat{\beta}_{T}, 0\right), a_{1} \leq \frac{x}{h^{i}} \leq 1
\end{array}\right. \\
& \text { Model } 4 m_{4}\left(x, d^{i}, h^{i}\right)=\left\{\begin{array}{l}
d^{i} \cdot \exp \left(\mu_{1}\left(\frac{x}{h^{i}} \mid \hat{\alpha}_{B}, \hat{\beta}_{B}, \hat{\sigma}_{B}, \frac{d_{0}^{i}}{d^{i}}\right)+\frac{1}{2} v_{1}\left(\frac{x}{h^{i}} \mid \hat{\beta}_{B}, \hat{\sigma}_{B}\right)\right), \frac{x}{h^{i}}<\frac{1.3}{h^{i}} \\
d^{i} \cdot \mu_{3}\left(\frac{x}{h^{i}} \mid \hat{\alpha}_{M}, \hat{\beta}_{M}, 1 .\right), \frac{1.3}{h^{i}} \leq \frac{x}{h^{i}}<a_{1} \\
d^{i} \cdot \mu_{3}\left(\frac{x}{h^{i}} \mid \hat{\alpha}_{T}, \hat{\beta}_{T}, 0\right), a_{1} \leq \frac{x}{h^{i}} \leq 1
\end{array},\right. \\
& \text { Model } 5 m_{5}\left(x, d^{i}, h^{i}\right)=\left\{\begin{array}{l}
d^{i} \cdot \exp \left(\mu_{1}\left(\frac{x}{h^{i}} \mid \hat{\alpha}_{B}, \hat{\beta}_{B}, \hat{\sigma}_{B}, \frac{d_{0}^{i}}{d^{i}}\right)+\frac{1}{2} v_{1}\left(\frac{x}{h^{i}} \mid \hat{\beta}_{B}, \hat{\sigma}_{B}\right)\right), \frac{x}{h^{i}} \leq a_{0} \\
d^{i} \cdot \mu_{3}\left(\frac{x}{h^{i}} \mid \hat{\alpha}_{M}, \hat{\beta}_{M}, \mu_{3}\left(a_{1} \mid \hat{\alpha}_{T}, \hat{\beta}_{T}, 0\right)\right), a_{0}<\frac{x}{h^{i}} \leq a_{1} \\
d^{i} \cdot \mu_{3}\left(\frac{x}{h^{i}} \mid \hat{\alpha}_{T}, \hat{\beta}_{T}, 0\right), a_{1}<\frac{x}{h^{i}} \leq 1
\end{array},\right. \\
& \text { Model } 6 m_{6}\left(x, d^{i}, h^{i}\right)=\left\{\begin{array}{l}
d^{i} \cdot \exp \left(\mu_{0}\left(\mu_{1}\left(\frac{x}{h^{i}} \mid \hat{\alpha}_{B}, \hat{\beta}_{B}, \hat{\sigma}_{B}, \hat{\gamma}\right)+\frac{1}{2} v_{1}\left(\frac{x}{h^{i}} \mid \hat{\beta}_{B}, \hat{\sigma}_{B}\right)\right)\right), \frac{x}{h^{i}}<a_{0} \\
d^{i} \cdot \mu_{3}\left(\frac{x}{h^{i}} \mid \hat{\alpha}_{M}, \hat{\beta}_{M}, 1 .\right), a_{0} \leq \frac{x}{h^{i}}<a_{1} \\
d^{i} \cdot \mu_{3}\left(\frac{x}{h^{i}} \mid \hat{\alpha}_{T}, \hat{\beta}_{T}, 0\right), a_{1} \leq \frac{x}{h^{i}} \leq 1
\end{array},\right. \\
& \text { Model } 7 m_{7}\left(x, d^{i}, h^{i}\right)=\left\{\begin{array}{l}
d^{i} \cdot \exp \left(\mu_{1}\left(\frac{x}{h^{i}} \mid \hat{\alpha}_{B}, \hat{\beta}_{B}, \hat{\sigma}_{B}, \frac{d_{0}^{i}}{d^{i}}\right)+\frac{1}{2} v_{1}\left(\frac{x}{h^{i}} \mid \hat{\beta}_{B}, \hat{\sigma}_{B}\right)\right), \frac{x}{h^{i}} \leq a_{0} \\
d^{i} \cdot \mu_{3}\left(\frac{x}{h^{i}} \mid \hat{\alpha}_{T}, \hat{\beta}_{T}, 0\right), a_{0}<\frac{x}{h^{i}} \leq 1
\end{array},\right. \\
& \text { Model } 8 m_{8}\left(x, d^{i}, h^{i}\right)=\left\{\begin{array}{l}
d^{i} \cdot \exp \left(\mu_{0}\left(\mu_{1}\left(\frac{x}{h^{i}} \mid \hat{\alpha}_{B}, \hat{\beta}_{B}, \hat{\sigma}_{B}, \hat{\gamma}\right)+\frac{1}{2} v_{1}\left(\frac{x}{h^{i}} \mid \hat{\beta}_{B}, \hat{\sigma}_{B}\right)\right)\right), \frac{x}{h^{i}} \leq a_{0} \\
d^{i} \cdot \mu_{3}\left(\frac{x}{h^{i}} \mid \hat{\alpha}_{T}, \hat{\beta}_{T}, 0\right), a_{0}<\frac{x}{h^{i}} \leq 1
\end{array} .\right.
\end{aligned}
$$

\subsection{Parameters Estimation}

Suppose that we observe the process of relative diameter $Y^{i}(x)$ in a discrete sense. Hence, the relative diameter $y^{i}\left(x_{j}^{i}\right)=y_{j}^{i}$ of the i-th tree is measured at $n_{i}+1$ discrete relative height points $\left(x_{0}^{i}, x_{1}^{i} \ldots, x_{n_{i}}^{i}\right) i=1, \ldots, M$. We let $y^{i}$ be the vector of the relative diameters for the $\mathrm{i}$-th tree, $\underline{y^{i}}=\left(y_{0}^{i}, y_{1}^{i}, \ldots, y_{n_{i}}^{i}\right)$, and $\underline{y}=\left(\underline{y}^{1}, \underline{y}^{2}, \ldots, \underline{y}^{M}\right)$ is the $n$-dimensional total relative diameter vector, $n=\sum_{i=1}^{M}\left(n_{i}+1\right)$. Therefore, we need to estimate fixed effect parameters $\mu_{0}, \sigma_{0}, \alpha_{1}, \beta_{1}, \sigma_{1}, \alpha_{2}, \sigma_{2}, \alpha_{3}, \beta_{3}, \sigma_{3}$ using all the data in $y$ simultaneously. In this study, we are interested in the maximum likelihood estimation of all fixed effect parameters.

Models 1 and 2 (Equations (13) and (14), 4 and 5 (Equations (16) and (17))), and 7 (Equation (19)) use a single tree-specific relative diameter $y_{0}^{i}$ (this known initial condition adds one more predictor variable expressed by the tree diameter measured at a height of $0 \mathrm{~m}$ ). Models 3 (Equation (15)), 6 (Equation (18)), and 8 (Equation (20)) use an additional log-normally distributed random variable, $\gamma \sim L N_{1}\left(\mu_{0} ; \sigma_{0}^{2}\right)$, which is the same for all stems. Models $1-8$ use a fixed relative diameter of 0 and a relative top height of 1 . The probability density functions of the relative diameter $Y^{i}\left(x_{j}^{i}\right), x_{j}^{i} \in[0 ; 1]$, $i=1, \ldots, M, j=0, \ldots, n_{i}$ for Models $1-8$ are defined, respectively, as follows:

$$
\begin{aligned}
& p_{1}\left(y_{j}^{i}, x_{j}^{i} \mid \theta_{1}, y_{j-1}^{i}, x_{j-1}^{i}\right)=\left\{\begin{array}{l}
p_{G}\left(y_{j}^{i}, x_{j}^{i} \mid \alpha_{B}, \beta_{B}, \sigma_{B}, y_{0}^{i}\right), 0<x_{j}^{i} \leq \frac{1.3}{h^{i}} \\
p_{g}\left(y_{j}^{i}, x_{j}^{i} \mid \alpha_{M}, \sigma_{M}, y_{j-1}^{i}, x_{j-1}^{i}\right), \frac{1.3}{h^{i}} \leq x_{j}^{i}<a_{1}, \\
p_{V}\left(y_{j}^{i}, x_{j}^{i} \mid \alpha_{T}, \beta_{T}, \sigma_{T}, 0,1\right), a_{1} \leq x_{j}^{i} \leq 1
\end{array}\right. \\
& \theta_{1}=\left\{\alpha_{B}, \beta_{B}, \sigma_{B}, \alpha_{M}, \sigma_{M}, \alpha_{T}, \beta_{T}, \sigma_{T}\right\}
\end{aligned}
$$




$$
\begin{aligned}
& p_{2}\left(y_{j}^{i}, x_{j}^{i} \mid \theta_{2}, y_{j+1}^{i}, x_{j+1}^{i}\right)=\left\{\begin{array}{l}
p_{G}\left(y_{j}^{i}, x_{j}^{i} \mid \alpha_{B}, \beta_{B}, \sigma_{B}, y_{0}^{i}\right), 0 \leq x_{j}^{i} \leq a_{0} \\
p_{g}\left(y_{j}^{i}, x_{j}^{i} \mid \alpha_{M}, \sigma_{M}, y_{j+1}^{i}, x_{j+1}^{i}\right), a_{0}<x_{j}^{i} \leq a_{1} \\
p_{V}\left(y_{j}^{i}, x_{j}^{i} \mid \alpha_{T}, \beta_{T}, \sigma_{T}, 0,1\right), a_{1}<x_{j}^{i} \leq 1
\end{array},\right. \\
& \theta_{2}=\left\{\alpha_{B}, \beta_{B}, \sigma_{B}, \alpha_{M}, \sigma_{M}, \alpha_{T}, \beta_{T}, \sigma_{T}\right\} \\
& p_{3}\left(y_{j}^{i}, x_{j}^{i} \mid \theta_{3}, y_{j-1}^{i}, x_{j-1}^{i}\right)=\left\{\begin{array}{l}
p_{G}\left(y_{j}^{i}, x_{j}^{i} \mid \alpha_{B}, \beta_{B}, \sigma_{B}, \gamma\right), 0 \leq x_{j}^{i}<\frac{1.3}{h^{i}} \\
p_{g}\left(y_{j}^{i}, x_{j}^{i} \mid \alpha_{M}, \sigma_{M}, y_{j+1}^{i}, x_{j+1}^{i}\right), \frac{1.3}{h^{i}} \leq x_{j}^{i}<a_{1}, \\
p_{V}\left(y_{j}^{i}, x_{j}^{i} \mid \alpha_{T}, \beta_{T}, \sigma_{T}, 0,1\right), a_{1} \leq x_{j}^{i} \leq 1
\end{array},\right. \\
& \theta_{3}=\left\{\mu_{0}, \sigma_{0}, \alpha_{B}, \beta_{B}, \sigma_{B}, \alpha_{M}, \sigma_{M}, \alpha_{T}, \beta_{T}, \sigma_{T}, \gamma\right\} \\
& p_{4}\left(y_{j}^{i}, x_{j}^{i} \mid \theta_{4}, y_{j-1}^{i}, x_{j-1}^{i}\right)=\left\{\begin{array}{l}
p_{G}\left(y_{j}^{i}, x_{j}^{i} \mid \alpha_{B}, \beta_{B}, \sigma_{B}, y_{0}^{i}\right), 0 \leq x_{j}^{i}<\frac{1.3}{h^{i}} \\
p_{V}\left(y_{j}^{i}, x_{j}^{i} \mid \alpha_{M}, \beta_{M}, \sigma_{M}, y_{j-1}^{i}, x_{j-1}^{i}\right), \frac{1.3}{h^{i}} \leq x_{j}^{i}<a_{1} \\
p_{V}\left(y_{j}^{i}, x_{j}^{i} \mid \alpha_{T}, \beta_{T}, \sigma_{T}, 0,1\right), a_{1} \leq x_{j}^{i} \leq 1
\end{array},\right. \\
& \theta_{4}=\left\{\alpha_{B}, \beta_{B}, \sigma_{B}, \alpha_{M}, \beta_{M}, \sigma_{M}, \alpha_{T}, \beta_{T}, \sigma_{T}\right\} \\
& p_{5}\left(y_{j}^{i}, x_{j}^{i} \mid \theta_{5}, y_{j+1}^{i}, x_{j+1}^{i}\right)=\left\{\begin{array}{l}
p_{G}\left(y_{j}^{i}, x_{j}^{i} \mid \alpha_{B}, \beta_{B}, \sigma_{B}, y_{0}^{i}\right), 0 \leq x_{j}^{i} \leq a_{0} \\
p_{V}\left(y_{j}^{i}, x_{j}^{i} \mid \alpha_{M}, \beta_{M}, \sigma_{M}, y_{j+1}^{i}, x_{j+1}^{i}\right), a_{0}<x_{j}^{i} \leq a_{1} \\
p_{V}\left(y_{j}^{i}, x_{j}^{i} \mid \alpha_{T}, \beta_{T}, \sigma_{T}, 0,1\right), a_{1}<x_{j}^{i} \leq 1
\end{array},\right. \\
& \theta_{5}=\left\{\alpha_{B}, \beta_{B}, \sigma_{B}, \alpha_{M}, \beta_{M}, \sigma_{M}, \alpha_{T}, \beta_{T}, \sigma_{T}\right\} \\
& p_{6}\left(y_{j}^{i}, x_{j}^{i} \mid \theta_{6}, y_{j-1}^{i}, x_{j-1}^{i}\right)=\left\{\begin{array}{l}
p_{G}\left(y_{j}^{i}, x_{j}^{i} \mid \alpha_{B}, \beta_{B}, \sigma_{B}, \gamma\right), 0 \leq x_{j}^{i}<\frac{1.3}{h^{i}} \\
p_{V}\left(y_{j}^{i}, x_{j}^{i} \mid \alpha_{M}, \beta_{M}, \sigma_{M}, y_{j+1}^{i}, x_{j+1}^{i}\right), \frac{1.3}{h^{i}} \leq x_{j}^{i}<a_{1}, \\
p_{V}\left(y_{j}^{i}, x_{j}^{i} \mid \alpha_{T}, \beta_{T}, \sigma_{T}, 0,1\right), a_{1} \leq x_{j}^{i} \leq 1
\end{array},\right. \\
& \theta_{6}=\left\{\mu_{0}, \sigma_{0}, \alpha_{B}, \beta_{B}, \sigma_{B}, \alpha_{M}, \beta_{M}, \sigma_{M}, \alpha_{T}, \beta_{T}, \sigma_{T}, \gamma\right\} \\
& p_{7}\left(y_{j}^{i}, x_{j}^{i} \mid \theta_{7}\right)=\left\{\begin{array}{l}
p_{G}\left(y_{j}^{i}, x_{j}^{i} \mid \alpha_{B}, \beta_{B}, \sigma_{B}, y_{0}^{i}\right), 0 \leq x_{j}^{i} \leq a_{0} \\
p_{V}\left(y_{j}^{i}, x_{j}^{i} \mid \alpha_{T}, \beta_{T}, \sigma_{T}, 0,1\right), a_{0}<x_{j}^{i} \leq 1
\end{array}, \theta_{7}=\left\{\alpha_{B}, \beta_{B}, \sigma_{B}, \alpha_{T}, \beta_{T}, \sigma_{T}\right\},\right. \\
& p_{8}\left(y_{j}^{i}, x_{j}^{i} \mid \theta_{8}\right)=\left\{\begin{array}{l}
p_{G}\left(y_{j}^{i}, x_{j}^{i} \mid \alpha_{B}, \beta_{B}, \sigma_{B}, \gamma\right), 0 \leq x_{j}^{i} \leq a_{0} \\
p_{V}\left(y_{j}^{i}, x_{j}^{i} \mid \alpha_{T}, \beta_{T}, \sigma_{T}, 0,1\right), a_{0}<x_{j}^{i} \leq 1
\end{array}, \theta_{8}=\left\{\mu_{0}, \sigma_{0}, \alpha_{B}, \beta_{B}, \sigma_{B}, \alpha_{T}, \beta_{T}, \sigma_{T}, \gamma\right\} .\right.
\end{aligned}
$$

In this paper, we apply the theory of a maximum likelihood procedure of the parameter estimates for stem taper in Models 1-8, as all models have closed form transition probability density functions defined by Equations (29)-(36). The fixed and mixed parameters estimation for discretely observed SDE is a complex problem and during the past decades it has attracted an attention of numerous researchers [30-32]. The log-likelihood functions are given, respectively, as follows:

$$
\begin{gathered}
L_{k}\left(\theta_{k}\right)=\sum_{i=1}^{M} \sum_{j=1}^{n_{i}} \ln \left(p_{k}\left(y_{j}^{i}, x_{j}^{i} \mid \theta_{k}, y_{j-1}^{i}, x_{j-1}^{i}\right)\right), k=1,3,4,6, \\
L_{k}\left(\theta_{k}\right)=\sum_{i=1}^{M} \sum_{j=1}^{n_{i}} \ln \left(p_{k}\left(y_{j}^{i}, x_{j}^{i} \mid \theta_{k}, y_{j+1}^{i}, x_{j+1}^{i}\right)\right), k=2,5,
\end{gathered}
$$




$$
L_{k}\left(\theta_{k}\right)=\sum_{i=1}^{M} \sum_{j=1}^{n_{i}} \ln \left(p_{k}\left(y_{j}^{i}, x_{j}^{i} \mid \theta_{8}\right)\right), k=7,8 .
$$

As the transition density functions are known, the maximum log-likelihood calculation and its maximization with respect to $\theta_{k}$, where $k=1-8$, for a given set of discretely observed datasets is straightforward. However, if $Y^{i}(0)=\gamma \sim L N_{1}\left(\mu_{0} ; \sigma_{0}^{2}\right)$, then there are two additional parameters that must be estimated. The estimation of parameters $\mu_{0}$ and $\sigma_{0}^{2}$ depends only on the measurement of each tree diameter at the height $h=0$ and does not influence the other parameters. The maximum likelihood estimations of $\mu_{0}$ and $\sigma_{0}^{2}$ for the log-normal distribution are given as follows:

$$
\begin{gathered}
\hat{\mu}=\frac{1}{M} \sum_{i=1}^{M} \ln \left(y_{0}^{i}\right), \\
\hat{\sigma}_{0}^{2}=\frac{1}{M} \sum_{i=1}^{M}\left(\ln \left(y_{0}^{i}\right)-\frac{1}{M} \sum_{i=1}^{M} \ln \left(y_{0}^{i}\right)\right)^{2} .
\end{gathered}
$$

\subsection{Statistical Measures}

The statistical measures of the stem taper equations for the diameter and the volume predictions include four statistical indices, namely, the coefficient of determination, $R^{2}=1-\frac{\sum_{i=1}^{n}\left(y_{i}-\hat{y}_{i}\right)^{2}}{\sum_{i=1}^{n}\left(y_{i}-\bar{y}\right)^{2}}$, the mean prediction error, $B=\frac{1}{n} \sum_{i=1}^{n}\left(y_{i}-\hat{y}_{i}\right)$, (percent prediction error, $\% B=\frac{1}{n} \sum_{i=1}^{n}\left(\frac{y_{i}-\hat{y}_{i}}{\bar{y}}\right)$ ), the mean absolute prediction error, $A B=\frac{1}{n} \sum_{i=1}^{n}\left|y_{i}-\hat{y}_{i}\right|$, (percent absolute prediction error, $\% A B=\frac{1}{n} \sum_{i=1}^{n} \frac{\left|y_{i}-\hat{y}_{i}\right|}{\bar{y}} \cdot 100$ ), and the root-mean-square error, $R M S E=\sqrt{\frac{1}{n-1} \sum_{i=1}^{n}\left(y_{i}-\hat{y}_{i}\right)^{2}}$, (percent root-mean-square error, \%RMSE $=$ $\left.\sqrt{\frac{1}{n-1} \sum_{i=1}^{n}\left(\frac{y_{i}-\hat{y}_{i}}{\bar{y}}\right)^{2}} \cdot 100\right)$. Here, $n$ is the total number of observations used to fit (validate) the model, and $y_{i}, \hat{y}_{i}$, and $\bar{y}$ are the observed, estimated, and average values, respectively, of the dependent variable (diameter, $d$, stem volume, $v$ ).

\subsection{Data}

Measurements for 3703 trees were used for the diameter at any specified height model and volume model analysis. All data were collected during 1979 to 2016 across the entire Lithuanian territory, except for Kuršiu Nerija National Park (latitude of $53.54-56.27^{\circ} \mathrm{N}$, longitude of 20.56-26.51 E, altitude of 10 to $293 \mathrm{~m}$ ). The mean temperatures vary from $-16.4{ }^{\circ} \mathrm{C}$ in winter to $22^{\circ} \mathrm{C}$ in summer. Precipitation is distributed throughout the year, although predominantly in summer, where the average is approximately $680 \mathrm{~mm}$ a year. Temporary circle test plots were placed in each of former 42 Lithuanian state forest enterprises in randomly selected clear-cutting areas. The diameter over bark of each stem in a plot was measured every $2 \mathrm{~m}$, starting from the diameter on the root collar, i.e., 1, 1.3, 3, 5, etc. All section measurements include of 47,413 data points. Diameters were measured to an accuracy of $1 \mathrm{~mm}$. The complete dataset was randomly divided into estimation and validation datasets. For all tree species, a random sample of 2617 trees (33,607 measurements) was selected for model estimation, and the remaining dataset of 1086 trees (13,806 measurements) was utilized for model validation (see Table 1). Summary statistics for the diameter over bark at breast height (d), total height (h), and volume (v) of all trees used for the fitting and validation of the models are presented in Table 1. The observed 
volume $\left(\mathrm{m}^{3}\right)$ of each stem was computed using a truncated cone formula, up to the last section, where the final apex was calculated as a cone [16]:

$$
V_{i}=\frac{\pi}{3 \cdot 40000}\left(\sum_{j=1}^{n_{i}-2}\left(d_{i j}^{2}+d_{i j+1}^{2}+d_{i j} \cdot d_{i j+1}\right) \cdot L_{i j}+d_{i n_{i}-1}^{2} \cdot L_{i n_{i}-1}\right),
$$

where $d_{i j}$ and $L_{i j}$ are the diameter $(\mathrm{cm})$ and length $(\mathrm{m})$, respectively, of the $\mathrm{j}$-th section $\mathrm{j}$ of the $\mathrm{i}$-th tree.

Table 1. Summary statistics of all measured stems. SD: standard deviation.

\begin{tabular}{|c|c|c|c|c|c|c|c|c|c|c|c|}
\hline \multirow[t]{2}{*}{ Species } & Data & $\begin{array}{l}\text { Number } \\
\text { of Stems }\end{array}$ & Min. & Max. & Mean & SD & $\begin{array}{l}\text { Number } \\
\text { of Stems }\end{array}$ & Min. & Max. & Mean & SD \\
\hline & \multicolumn{6}{|c|}{ Estimation } & \multicolumn{5}{|c|}{ Validation } \\
\hline \multirow{3}{*}{ Oak } & $\mathrm{d}(\mathrm{cm})$ & 101 & 7.0 & 49.6 & 21.33 & 8.98 & 45 & 6.0 & 43.0 & 24.40 & 8.05 \\
\hline & $h(m)$ & 101 & 9.6 & 29.9 & 21.60 & 4.24 & 45 & 11.3 & 29.0 & 22.68 & 3.82 \\
\hline & $\mathrm{v}\left(\mathrm{m}^{3}\right)$ & 101 & 0.026 & 2.801 & 0.490 & 0.481 & 45 & 0.020 & 2.047 & 0.591 & 0.424 \\
\hline \multirow{3}{*}{ Ash } & $\mathrm{d}(\mathrm{cm})$ & 27 & 8.0 & 29.0 & 18.33 & 4.60 & 11 & 10 & 28 & 19.21 & 5.96 \\
\hline & h (m) & 27 & 11.2 & 25.7 & 19.29 & 2.98 & 11 & 13 & 25.1 & 19.17 & 3.12 \\
\hline & $\mathrm{v}\left(\mathrm{m}^{3}\right)$ & 27 & 0.029 & 0.831 & 0.273 & 0.175 & 11 & 0.052 & 0.622 & 0.295 & 0.183 \\
\hline \multirow{3}{*}{ Birch } & $\mathrm{d}(\mathrm{cm})$ & 230 & 7.6 & 51.2 & 23.26 & 9.04 & 103 & 8.0 & 49.3 & 22.78 & 8.65 \\
\hline & $h(m)$ & 230 & 10.2 & 31.9 & 21.39 & 4.58 & 103 & 8.4 & 31.3 & 21.37 & 4.26 \\
\hline & $\mathrm{v}\left(\mathrm{m}^{3}\right)$ & 230 & 0.027 & 2.583 & 0.504 & 0.439 & 103 & 0.027 & 2.190 & 0.470 & 0.391 \\
\hline \multirow{3}{*}{$\begin{array}{l}\text { Black } \\
\text { alder }\end{array}$} & $\mathrm{d}(\mathrm{cm})$ & 136 & 8.0 & 39.9 & 22.74 & 5.65 & 61 & 12.0 & 33.5 & 22.02 & 5.03 \\
\hline & h (m) & 136 & 8.5 & 27.6 & 20.59 & 3.57 & 61 & 13.2 & 27.4 & 20.66 & 3.42 \\
\hline & $\mathrm{v}\left(\mathrm{m}^{3}\right)$ & 136 & 0.019 & 1.806 & 0.467 & 0.282 & 61 & 0.077 & 0.883 & 0.426 & 0.215 \\
\hline \multirow{3}{*}{$\begin{array}{l}\text { White } \\
\text { alder }\end{array}$} & $\mathrm{d}(\mathrm{cm})$ & 16 & 7.7 & 8.0 & 17.64 & 5.55 & 6 & 12.0 & 24.2 & 17.68 & 4.26 \\
\hline & h (m) & 16 & 11.3 & 23.4 & 17.26 & 3.10 & 6 & 15.4 & 20.2 & 17.22 & 1.72 \\
\hline & $\mathrm{v}\left(\mathrm{m}^{3}\right)$ & 16 & 0.029 & 0.693 & 0.240 & 0.166 & 6 & 0.097 & 0.403 & 0.221 & 0.112 \\
\hline \multirow{3}{*}{ Aspen } & $\mathrm{d}(\mathrm{cm})$ & 102 & 7.0 & 49.6 & 21.34 & 8.94 & 44 & 6.0 & 43.0 & 24.45 & 8.13 \\
\hline & h (m) & 102 & 9.6 & 29.9 & 21.65 & 4.24 & 44 & 11.3 & 29.0 & 22.61 & 3.83 \\
\hline & $\mathrm{v}\left(\mathrm{m}^{3}\right)$ & 102 & 0.026 & 2.801 & 0.489 & 0.479 & 44 & 0.020 & 2.049 & 0.595 & 0.429 \\
\hline \multirow{3}{*}{ Pine } & $\mathrm{d}(\mathrm{cm})$ & 1344 & 5.0 & 58.4 & 25.25 & 9.86 & 567 & 3.8 & 58.5 & 23.95 & 9.87 \\
\hline & $h(m)$ & 1344 & 4.5 & 35.2 & 20.85 & 5.23 & 567 & 3.8 & 33.5 & 20.14 & 5.50 \\
\hline & $\mathrm{v}\left(\mathrm{m}^{3}\right)$ & 1344 & 0.006 & 3.129 & 0.626 & 0.562 & 567 & 0.003 & 3.398 & 0.570 & 0.563 \\
\hline \multirow{3}{*}{ Spruce } & $\mathrm{d}(\mathrm{cm})$ & 661 & 7.9 & 52.4 & 23.14 & 8.78 & 249 & 8.0 & 49.8 & 23.34 & 8.31 \\
\hline & $h(m)$ & 661 & 7.0 & 32.7 & 20.92 & 5.54 & 249 & 7.5 & 33.8 & 21.12 & 5.23 \\
\hline & $v\left(m^{3}\right)$ & 661 & 0.021 & 2.737 & 0.571 & 0.486 & 249 & 0.018 & 2.994 & 0.571 & 0.423 \\
\hline \multicolumn{2}{|c|}{ Number of stems } & 2617 & & & & & 1086 & & & & \\
\hline
\end{tabular}

\subsection{Regression Stem Taper Models}

Forest statisticians have developed multiple advanced regression models for describing tree stem tapers using more and more complex nonlinear functions. The newly developed SDE models for stem tapers will be compared with a well-known and frequently used stem taper model [14-16].

The segmented polynomial model published by Burkhart [14].

Model $9 \frac{d^{2}}{D^{2}}=\beta_{1}(z-1) \beta_{1}(z-1)+\beta_{2}\left(z^{2}-1\right)+\beta_{3}\left(\beta_{5}-z\right) I_{1}\left(\beta_{5}-z\right)+\beta_{4}\left(\beta_{6}-z\right)^{2} I_{2}\left(\beta_{6}-z\right)$, 
where $d$ is the diameter at any particular height $h, D$ is the diameter at breast height, $H$ is the total tree height, $z$ is the relative height $\left(z=\frac{h}{H}\right), \beta_{5}$ and $\beta_{6}$ are the joining points of three segments, and $\beta_{1}-\beta_{6}$ are the unknown parameters to be estimated. Additionally,

$$
I_{i}\left(\beta_{i}-z\right)=\left\{\begin{array}{l}
1, \beta_{i}-z \geq 0 \\
0, \beta_{i}-z<0
\end{array} .\right.
$$

A generalized q-exponential model of stem taper published by Rupšys and Petrauskas et al. [16].

$$
\text { Model 10 } d=\beta_{1}+\beta_{2} D^{\beta_{3}}\left(1-z^{8}+0.0001\right)^{\beta_{4}}\left\{\begin{array}{l}
\beta_{5}(z-1)+\beta_{6}\left(z^{2}-1\right), \text { if } z \geq \alpha \\
{\left[\beta_{7}-\beta_{8}\left(1-\exp \left(\left(1-\beta_{9}\right) z\right)\right)\right]_{+}^{\frac{1}{1-\beta_{9}}}}
\end{array},\right.
$$

where $\alpha$ is the joining point of two segments, $\beta_{1}-\beta_{9}$ are the unknown parameters to be estimated, and

$$
[a]_{+}=\left\{\begin{array}{c}
a, i f a \geq 0 \\
0, i f a<0
\end{array} .\right.
$$

The stem taper model published by Kozak [15].

$$
\text { Model } 11 d=\beta_{1} D^{\beta_{2}} H^{\beta_{3}}\left(\frac{1-\sqrt[3]{z}+0.0001}{1-\sqrt[3]{\frac{1.3}{H}}}\right)^{\beta_{4} z^{4}+\frac{\beta_{5}}{\exp (D / H)}+\beta_{6}\left(\frac{1-\sqrt[3]{z}}{1-\sqrt[3]{\frac{1.3}{H}}}\right)^{0.1}+\frac{\beta_{7}}{D}+\beta_{8} H^{1-\sqrt[3]{z}}+\beta_{9}\left(\frac{1-\sqrt[3]{z}}{1-\sqrt[3]{\frac{1.3}{H}}}\right)},
$$

where $\beta_{1}-\beta_{9}$ are the unknown parameters to be estimated.

\section{Results}

Various methods of advanced modeling are used to describe the evolution of stem profiles. Nowadays, the available computational power has substantially increased, so it becomes feasible to use more advanced SDE models and to introduce some kind of noise in the stem taper form. In this study, the developed models are nondeterministic models that consider both the symmetric and asymmetric diffusions of stem diameter at a particular height [21]. Multiple regression models that describe stem tapers have been proposed, traditionally incorporating the diameter at breast height and tree height as the independent variables [14-19] or using additional independent variables such as the density [18], crown ratio [33], and many more [34,35]. The newly developed SDE models are affected by the probability density function of the relative diameter, which changes among the relative heights. Another characteristic of our proposed SDE models is that they take into account a 'noise' term, defined by a standard Brownian motion, which in turn leads to a solution that is a diffusion process. Different from other methods, we explore a variety of characteristics of diffusion processes, including transition probability density functions, mean trends, and variance trends. SDE models can be applied to express the variation in individual stem tapers by introducing random effects. However, the efficiency of the random effects is largely dependent on the amount of additional information, which involves the measurement of tree diameters at specified heights in standing trees.

This study focuses on two new SDE stem taper modeling techniques, namely, using the diameter at breast height, total tree height, and diameter at stem butt (at the height of $0.0 \mathrm{~cm}$ ) as incorporated independent (predictor) variables. Models 1, 2, 4, 5, and 7 feature this technique. The second technique is using the diameter at breast height and total tree height as incorporated predictor variables. Models 3,6 , and 8 use this technique. The results of both the SDE tree taper modeling techniques are compared with the traditionally used regression taper models, shown in Models 9, 10, and 11. The efficiencies of the statistically estimated stem taper equations for volume predictions are examined. For both purposes, efficient diameter and volume predictions are not necessary and can be simultaneously determined. 


\subsection{Parameter Estimates}

The methodology of optimal parameter estimation using SDEs generally considers the fitting of the parameters that maximize the log-likelihood of the observed discrete dataset, as an exact form expression for the log-likelihood function is available (see Equations (37)-(39)). The fixed effect parameters of the SDE stem tapers in Models 1-8 were estimated utilizing the maximum likelihood methodology developed in Section 2.3. The parameters of nonlinear regression in Models 9-11 were estimated by the least-square methodology. The lower joint point, $\mathrm{a}_{0}$, for the SDEs models is determined by the initial condition that the relative tree diameter is equal to 1 at a relative height $1.3 / \mathrm{h}$. In order to examine upper joint point, $\mathrm{a}_{1}$, the maximum likelihood procedure was simulated by fixing the lower joint point at $1.3 / \mathrm{h}$ and varying the upper joint point. For the regression model 10, the joint point, $\mathrm{a}_{0}$, was examined by generating taper equation and assigning value according to interval [0.11; 0.75]. Parameters estimates were computed using the estimation dataset. For all of fitted models, the parameters estimates were obtained using the "Statistics" and "VectorCalculus" packages in the Maple symbolic algebra system [36].

\subsection{Stem Taper and Volume Models}

A comparison of all fitted models was undertaken using the validation dataset. In order to be an applicable taper model, a model needs to predict diameter measurements at a particular height in a stem highly accurately. Diameter predictions at any specified height were computed for all fitted stem taper models, and the stem volume predictions were computed by integration-derived stem taper curves using the validation dataset for each species group. To compare all fitted taper and volume models, we used statistical measures (i.e., the coefficient of determination, mean prediction error (percent prediction error, \%), mean absolute prediction error (percent absolute prediction error, \%), and root-mean-square error (percent root-mean-square error, \%), including analysis of the visual tests of the residuals and the simplicity. Quantitative assessment of models performance will rely on statistical measures including four basic features: the bias, which indicates that the predictions are systematically too high or too low (underperformance or overperformance); the absolute bias, which describes the typical magnitude of the residuals (does not indicate underperformance or overperformance); the root mean squared error of differences between observed and predicted values, which indicates standard deviation of the unexplained variance; and the coefficient of determination, which informs on the proportion of the total variability explained by the model. The root-mean-square error, in a statistical sense, normally takes precedence over the other errors, where it is known as the standard error of the regression in regression analysis or as the estimated white noise standard deviation in stochastic processes.

The main problem of the comparison of the stem taper models is measuring the predictive ability to accurately predict the diameter and volume of new tree stems. Statistical measures calculated for the estimation dataset evaluate how well the stem taper model fits the estimation dataset for which it was developed, and, for the validation dataset, confirm how valid the stem taper model is in predicting diameters at any specified height and tree volumes for new dataset [37]. The decrease of statistical measures for the validation dataset in this study was small, so we can conclude that the used stem taper models can be used to predict stem diameters at any specified height, as well as stem volumes.

Statistical measures for the prediction of the diameter and volume using stem diameter measurements from the validation dataset for all species group are presented in Tables 2 and 3, respectively. In most cases (models and species groups), the stem diameters and volumes have been slightly overpredicted. The black alder species group models produced the best root-mean-square error percent values, namely, $6.62 \%$ to $8.93 \%$ and $7.40 \%$ to $8.06 \%$ for the diameter and volume predictions, respectively. The birch species group models produced the worst root-mean-square error percent values, namely, $10.42 \%$ to $13.04 \%$ and $17.70 \%$ to $27.34 \%$ for the diameter and volume predictions, respectively. 
Table 2. Statistical measures for all of the validated stem diameter models *

\begin{tabular}{|c|c|c|c|c|c|c|c|c|c|c|c|c|}
\hline Model & $\begin{array}{c}\text { B } \\
(\%)\end{array}$ & $\begin{array}{l}\mathrm{AB} \\
(\%)\end{array}$ & $\begin{array}{c}\text { RMSE } \\
(\%)\end{array}$ & $\mathbf{R}^{2}$ & $\begin{array}{c}\text { B } \\
(\%)\end{array}$ & $\begin{array}{l}\mathrm{AB} \\
(\%)\end{array}$ & $\begin{array}{c}\text { RMSE } \\
(\%)\end{array}$ & $\mathbf{R}^{2}$ & $\begin{array}{c}\text { B } \\
(\%)\end{array}$ & $\begin{array}{l}\text { AB } \\
(\%)\end{array}$ & $\begin{array}{c}\text { RMSE } \\
(\%)\end{array}$ & $\mathbf{R}^{2}$ \\
\hline & \multicolumn{4}{|c|}{ Oak } & \multicolumn{4}{|c|}{ Ash } & \multicolumn{4}{|c|}{ Birch } \\
\hline 1 & $\begin{array}{l}-0.217 \\
(-1.31) \\
\end{array}$ & $\begin{array}{l}0.832 \\
(5.01) \\
\end{array}$ & $\begin{array}{l}1.269 \\
(7.65)\end{array}$ & 0.986 & $\begin{array}{l}-0.059 \\
(-0.45) \\
\end{array}$ & $\begin{array}{l}0.706 \\
(5.36) \\
\end{array}$ & $\begin{array}{l}1.057 \\
(8.03) \\
\end{array}$ & 0.985 & $\begin{array}{l}-0.382 \\
(-2.39) \\
\end{array}$ & $\begin{array}{l}1.047 \\
(6.56) \\
\end{array}$ & $\begin{array}{c}1.747 \\
(10.95)\end{array}$ & 0.970 \\
\hline 2 & $\begin{array}{l}-0.262 \\
(-1.58)\end{array}$ & $\begin{array}{l}0.833 \\
(5.02)\end{array}$ & $\begin{array}{l}1.259 \\
(7.59)\end{array}$ & 0.986 & $\begin{array}{l}0.033 \\
(0.25)\end{array}$ & $\begin{array}{l}0.712 \\
(5.41)\end{array}$ & $\begin{array}{c}1.101 \\
(8.36)\end{array}$ & 0.984 & $\begin{array}{l}0.095 \\
(0.60)\end{array}$ & $\begin{array}{l}1.120 \\
(7.02)\end{array}$ & $\begin{array}{c}1.676 \\
(10.50)\end{array}$ & 0.972 \\
\hline 3 & $\begin{array}{l}-0.280 \\
(-1.69)\end{array}$ & $\begin{array}{l}1.010 \\
(6.09)\end{array}$ & $\begin{array}{l}1.524 \\
(9.19)\end{array}$ & 0.979 & $\begin{array}{l}0.032 \\
(0.25)\end{array}$ & $\begin{array}{l}0.936 \\
(7.11)\end{array}$ & $\begin{array}{c}1.469 \\
(11.16)\end{array}$ & 0.962 & $\begin{array}{l}-0.422 \\
(-2.64)\end{array}$ & $\begin{array}{l}1.263 \\
(7.92)\end{array}$ & $\begin{array}{c}2.006 \\
(12.57)\end{array}$ & 0.960 \\
\hline 4 & $\begin{array}{l}-0.610 \\
(-3.68)\end{array}$ & $\begin{array}{l}0.952 \\
(5.74) \\
\end{array}$ & $\begin{array}{l}1.320 \\
(7.96)\end{array}$ & 0.984 & $\begin{array}{l}0.015 \\
(0.12)\end{array}$ & $\begin{array}{l}0.728 \\
(5.53) \\
\end{array}$ & $\begin{array}{l}1.077 \\
(8.18)\end{array}$ & 0.985 & $\begin{array}{l}-0.437 \\
(-2.74)\end{array}$ & $\begin{array}{l}1.167 \\
(7.31) \\
\end{array}$ & $\begin{array}{c}1.924 \\
(12.06)\end{array}$ & 0.963 \\
\hline 5 & $\begin{array}{l}-0.442 \\
(-2.66) \\
\end{array}$ & $\begin{array}{r}0.855 \\
(5.15) \\
\end{array}$ & $\begin{array}{l}1.236 \\
(7.45)\end{array}$ & 0.986 & $\begin{array}{l}-0.156 \\
(-1.19)\end{array}$ & $\begin{array}{l}0.717 \\
(5.44) \\
\end{array}$ & $\begin{array}{l}1.054 \\
(8.01)\end{array}$ & 0.985 & $\begin{array}{l}-0.143 \\
(-0.89)\end{array}$ & $\begin{array}{l}1.025 \\
(6.42) \\
\end{array}$ & $\begin{array}{c}1.663 \\
(10.42) \\
\end{array}$ & 0.972 \\
\hline 6 & $\begin{array}{l}-0.394 \\
(-2.38)\end{array}$ & $\begin{array}{l}1.021 \\
(6.16)\end{array}$ & $\begin{array}{l}1.496 \\
(9.02)\end{array}$ & 0.980 & $\begin{array}{l}0.137 \\
(1.04)\end{array}$ & $\begin{array}{l}0.907 \\
(6.69)\end{array}$ & $\begin{array}{c}1.427 \\
(10.84)\end{array}$ & 0.973 & $\begin{array}{l}-0.112 \\
(-0.70)\end{array}$ & $\begin{array}{l}1.232 \\
(7.72)\end{array}$ & $\begin{array}{c}1.918 \\
(12.02)\end{array}$ & 0.963 \\
\hline 7 & $\begin{array}{l}-0.388 \\
(-2.34)\end{array}$ & $\begin{array}{l}0.846 \\
(5.10)\end{array}$ & $\begin{array}{l}1.242 \\
(7.49)\end{array}$ & 0.986 & $\begin{array}{l}-0.181 \\
(-1.38)\end{array}$ & $\begin{array}{l}0.732 \\
(5.56)\end{array}$ & $\begin{array}{l}1.064 \\
(8.08)\end{array}$ & 0.985 & $\begin{array}{l}-0.266 \\
(-1.67)\end{array}$ & $\begin{array}{l}1.026 \\
(6.43)\end{array}$ & $\begin{array}{c}1.690 \\
(10.59)\end{array}$ & 0.971 \\
\hline 8 & $\begin{array}{l}-0.396 \\
(-2.39)\end{array}$ & $\begin{array}{l}1.037 \\
(6.25) \\
\end{array}$ & $\begin{array}{l}1.520 \\
(9.16)\end{array}$ & 0.979 & $\begin{array}{l}0.079 \\
(0.60) \\
\end{array}$ & $\begin{array}{l}1.032 \\
(7.84) \\
\end{array}$ & $\begin{array}{c}1.700 \\
(12.92)\end{array}$ & 0.961 & $\begin{array}{c}-0.286 \\
(1.79)\end{array}$ & $\begin{array}{l}1.236 \\
(7.75) \\
\end{array}$ & $\begin{array}{c}1.948 \\
(12.21)\end{array}$ & 0.962 \\
\hline 9 & $\begin{array}{c}-0.503 \\
(3.02)\end{array}$ & $\begin{array}{l}1.071 \\
(6.46)\end{array}$ & $\begin{array}{l}1.548 \\
(9.33)\end{array}$ & 0.978 & $\begin{array}{l}-0.166 \\
(-1.26)\end{array}$ & $\begin{array}{l}0.987 \\
(7.50)\end{array}$ & $\begin{array}{c}1.491 \\
(11.33)\end{array}$ & 0.970 & $\begin{array}{l}-0.391 \\
(-2.45)\end{array}$ & $\begin{array}{l}1.364 \\
(8.55)\end{array}$ & $\begin{array}{c}2.081 \\
(13.04)\end{array}$ & 0.957 \\
\hline 10 & $\begin{array}{l}-0.249 \\
(-1.50)\end{array}$ & $\begin{array}{l}1.012 \\
(6.10)\end{array}$ & $\begin{array}{l}1.485 \\
(8.95)\end{array}$ & 0.980 & $\begin{array}{l}-0.112 \\
(-0.85)\end{array}$ & $\begin{array}{l}1.025 \\
(7.78)\end{array}$ & $\begin{array}{c}1.511 \\
(11.48)\end{array}$ & 0.970 & $\begin{array}{l}-0.107 \\
(-0.67)\end{array}$ & $\begin{array}{l}1.196 \\
(7.49)\end{array}$ & $\begin{array}{c}1.752 \\
(10.98)\end{array}$ & 0.969 \\
\hline \multirow[t]{2}{*}{11} & $\begin{array}{l}-0.253 \\
(-1.53)\end{array}$ & $\begin{array}{l}1.006 \\
(6.07) \\
\end{array}$ & $\begin{array}{l}1.519 \\
(9.16)\end{array}$ & 0.979 & $\begin{array}{l}0.080 \\
(0.61) \\
\end{array}$ & $\begin{array}{l}1.031 \\
(7.83) \\
\end{array}$ & $\begin{array}{c}1.637 \\
(12.44)\end{array}$ & 0.964 & $\begin{array}{l}-0.139 \\
(-0.87)\end{array}$ & $\begin{array}{l}1.151 \\
(7.21) \\
\end{array}$ & $\begin{array}{c}1.734 \\
(\mathbf{1 0 . 8 6})\end{array}$ & 0.970 \\
\hline & \multicolumn{4}{|c|}{ Black alder } & \multicolumn{4}{|c|}{ White alder } & \multicolumn{4}{|c|}{ Aspen } \\
\hline 1 & $\begin{array}{l}-0.005 \\
(-0.03)\end{array}$ & $\begin{array}{l}0.673 \\
(4.33)\end{array}$ & $\begin{array}{l}1.043 \\
(6.71)\end{array}$ & 0.985 & $\begin{array}{l}-0.150 \\
(-1.24)\end{array}$ & $\begin{array}{l}0.688 \\
(5.68)\end{array}$ & $\begin{array}{l}0.990 \\
(8.18)\end{array}$ & 0.983 & $\begin{array}{l}-0.344 \\
(-2.08)\end{array}$ & $\begin{array}{l}0.846 \\
(5.11)\end{array}$ & $\begin{array}{l}1.247 \\
(7.54)\end{array}$ & 0.986 \\
\hline 2 & $\begin{array}{l}0.147 \\
(0.94) \\
\end{array}$ & $\begin{array}{l}0.720 \\
(4.63) \\
\end{array}$ & $\begin{array}{l}1.092 \\
(7.02)\end{array}$ & 0.984 & $\begin{array}{l}-0.164 \\
(-1.36)\end{array}$ & $\begin{array}{l}0.704 \\
(5.82) \\
\end{array}$ & $\begin{array}{l}1.020 \\
(8.42)\end{array}$ & 0.982 & $\begin{array}{l}-0.241 \\
(-1.46)\end{array}$ & $\begin{array}{l}0.833 \\
(5.04) \\
\end{array}$ & $\begin{array}{l}1.258 \\
(7.60) \\
\end{array}$ & 0.986 \\
\hline 3 & $\begin{array}{l}-0.051 \\
(-0.33) \\
\end{array}$ & $\begin{array}{l}0.799 \\
(5.14) \\
\end{array}$ & $\begin{array}{l}1.256 \\
(8.08)\end{array}$ & 0.978 & $\begin{array}{l}-0.102 \\
(-0.84)\end{array}$ & $\begin{array}{l}0.808 \\
(6.68) \\
\end{array}$ & $\begin{array}{l}1.176 \\
(9.71)\end{array}$ & 0.976 & $\begin{array}{l}-0.343 \\
(-2.07)\end{array}$ & $\begin{array}{l}1.026 \\
(6.20) \\
\end{array}$ & $\begin{array}{l}1.536 \\
(9.28) \\
\end{array}$ & 0.979 \\
\hline 4 & $\begin{array}{l}0.064 \\
(0.41)\end{array}$ & $\begin{array}{l}0.714 \\
(4.59)\end{array}$ & $\begin{array}{l}1.098 \\
(7.06)\end{array}$ & 0.983 & $\begin{array}{l}0.099 \\
(0.82)\end{array}$ & $\begin{array}{l}0.648 \\
(5.35)\end{array}$ & $\begin{array}{l}1.005 \\
(8.30)\end{array}$ & 0.983 & $\begin{array}{l}-0.095 \\
(-0.57)\end{array}$ & $\begin{array}{l}0.803 \\
(4.86)\end{array}$ & $\begin{array}{l}1.228 \\
(7.42)\end{array}$ & 0.986 \\
\hline 5 & $\begin{array}{l}-0.060 \\
(-0.38)\end{array}$ & $\begin{array}{l}0.663 \\
(4.27)\end{array}$ & $\begin{array}{l}1.030 \\
(6.62)\end{array}$ & 0.985 & $\begin{array}{l}-0.192 \\
(-1.59)\end{array}$ & $\begin{array}{l}0.724 \\
(5.99)\end{array}$ & $\begin{array}{l}1.008 \\
(8.33)\end{array}$ & 0.983 & $\begin{array}{l}-0.405 \\
(-2.45)\end{array}$ & $\begin{array}{l}0.848 \\
(5.13)\end{array}$ & $\begin{array}{l}1.230 \\
(7.44)\end{array}$ & 0.986 \\
\hline
\end{tabular}


Table 2. Cont.

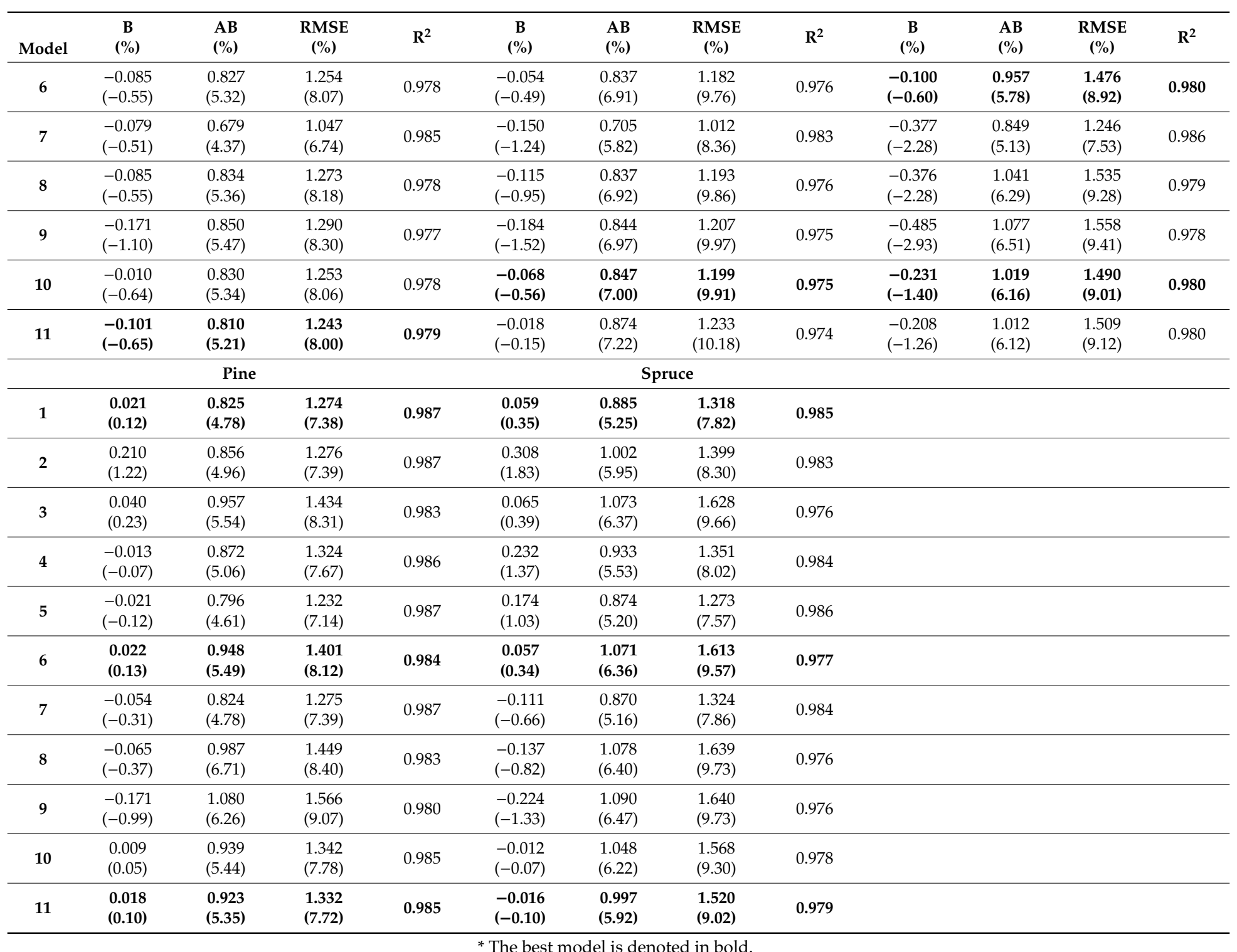


Table 3. Statistical measures for all of the validated volume models *.

\begin{tabular}{|c|c|c|c|c|c|c|c|c|c|c|c|c|}
\hline \multirow[t]{2}{*}{ Model } & $\begin{array}{c}\text { B } \\
(\%)\end{array}$ & $\begin{array}{l}A B \\
(\%)\end{array}$ & $\begin{array}{c}\text { RMSE } \\
(\%)\end{array}$ & $R^{2}$ & $\begin{array}{c}\text { B } \\
(\%)\end{array}$ & $\begin{array}{l}A B \\
(\%)\end{array}$ & $\begin{array}{c}\text { RMSE } \\
(\%)\end{array}$ & $\mathbf{R}^{2}$ & $\begin{array}{c}\text { B } \\
(\%)\end{array}$ & $\begin{array}{l}A B \\
(\%)\end{array}$ & $\begin{array}{c}\text { RMSE } \\
(\%)\end{array}$ & $\mathbf{R}^{2}$ \\
\hline & \multicolumn{4}{|c|}{ Oak } & \multicolumn{4}{|c|}{ Ash } & \multicolumn{4}{|c|}{ Birch } \\
\hline 1 & $\begin{array}{l}-0.002 \\
(-0.43)\end{array}$ & $\begin{array}{l}0.040 \\
(6.79) \\
\end{array}$ & $\begin{array}{c}0.064 \\
(10.94) \\
\end{array}$ & 0.977 & $\begin{array}{l}-0.002 \\
(-0.72)\end{array}$ & $\begin{array}{l}0.023 \\
(7.76) \\
\end{array}$ & $\begin{array}{c}0.030 \\
(10.25) \\
\end{array}$ & 0.975 & $\begin{array}{l}-0.035 \\
(-7.46) \\
\end{array}$ & $\begin{array}{c}0.060 \\
(12.89) \\
\end{array}$ & $\begin{array}{c}0.111 \\
(23.67) \\
\end{array}$ & 0.920 \\
\hline 2 & $\begin{array}{l}-0.006 \\
(-1.10)\end{array}$ & $\begin{array}{l}0.039 \\
(6.67)\end{array}$ & $\begin{array}{c}0.061 \\
(10.34)\end{array}$ & 0.980 & $\begin{array}{l}0.002 \\
(0.74)\end{array}$ & $\begin{array}{l}0.021 \\
(7.26)\end{array}$ & $\begin{array}{c}0.052 \\
(17.63)\end{array}$ & 0.926 & $\begin{array}{l}0.006 \\
(1.29)\end{array}$ & $\begin{array}{c}0.058 \\
(12.24)\end{array}$ & $\begin{array}{c}0.090 \\
(19.07)\end{array}$ & 0.948 \\
\hline 3 & $\begin{array}{c}-0.009 \\
(1.49)\end{array}$ & $\begin{array}{l}0.042 \\
(7.04)\end{array}$ & $\begin{array}{c}0.060 \\
(10.08)\end{array}$ & 0.981 & $\begin{array}{l}-0.000 \\
(-0.10)\end{array}$ & $\begin{array}{l}0.020 \\
(6.93)\end{array}$ & $\begin{array}{l}0.026 \\
(8.65)\end{array}$ & 0.982 & $\begin{array}{l}-0.039 \\
(-8.29)\end{array}$ & $\begin{array}{c}0.064 \\
(13.71)\end{array}$ & $\begin{array}{c}0.120 \\
(25.57)\end{array}$ & 0.906 \\
\hline 4 & $\begin{array}{l}-0.041 \\
(-6.99)\end{array}$ & $\begin{array}{l}0.052 \\
(8.80) \\
\end{array}$ & $\begin{array}{c}0.065 \\
(10.97)\end{array}$ & 0.977 & $\begin{array}{c}0.0001 \\
(0.02)\end{array}$ & $\begin{array}{l}0.022 \\
(7.73) \\
\end{array}$ & $\begin{array}{c}0.030 \\
(10.18)\end{array}$ & 0.975 & $\begin{array}{l}-0.045 \\
(-9.56)\end{array}$ & $\begin{array}{c}0.069 \\
(14.73)\end{array}$ & $\begin{array}{c}0.129 \\
(27.34)\end{array}$ & 0.893 \\
\hline 5 & $\begin{array}{l}-0.025 \\
(-4.23)\end{array}$ & $\begin{array}{l}0.043 \\
(7.29) \\
\end{array}$ & $\begin{array}{c}0.061 \\
(10.39)\end{array}$ & 0.980 & $\begin{array}{l}-0.008 \\
(-2.69)\end{array}$ & $\begin{array}{l}0.023 \\
(7.87) \\
\end{array}$ & $\begin{array}{c}0.030 \\
(10.44)\end{array}$ & 0.974 & $\begin{array}{l}-0.015 \\
(-3.22) \\
\end{array}$ & $\begin{array}{c}0.058 \\
(12.46)\end{array}$ & $\begin{array}{c}0.101 \\
(21.60)\end{array}$ & 0.933 \\
\hline 6 & $\begin{array}{l}-0.021 \\
(-3.51)\end{array}$ & $\begin{array}{l}0.043 \\
(7.35) \\
\end{array}$ & $\begin{array}{l}0.058 \\
(9.78)\end{array}$ & 0.982 & $\begin{array}{l}0.003 \\
(1.18)\end{array}$ & $\begin{array}{l}0.020 \\
(6.73) \\
\end{array}$ & $\begin{array}{l}0.025 \\
(8.30) \\
\end{array}$ & 0.984 & $\begin{array}{l}-0.014 \\
(-2.99)\end{array}$ & $\begin{array}{c}0.061 \\
(12.92)\end{array}$ & $\begin{array}{c}0.107 \\
(22.66)\end{array}$ & 0.926 \\
\hline 7 & $\begin{array}{l}-0.018 \\
(-3.08)\end{array}$ & $\begin{array}{l}0.041 \\
(6.98)\end{array}$ & $\begin{array}{c}0.059 \\
(10.06)\end{array}$ & 0.981 & $\begin{array}{l}-0.009 \\
(-2.90)\end{array}$ & $\begin{array}{l}0.023 \\
(7.86)\end{array}$ & $\begin{array}{c}0.031 \\
(10.50)\end{array}$ & 0.974 & $\begin{array}{l}-0.025 \\
(-5.31)\end{array}$ & $\begin{array}{c}0.059 \\
(12.66)\end{array}$ & $\begin{array}{c}0.107 \\
(22.74)\end{array}$ & 0.923 \\
\hline 8 & $\begin{array}{l}-0.019 \\
(-3.23)\end{array}$ & $\begin{array}{l}0.043 \\
(7.24)\end{array}$ & $\begin{array}{l}0.057 \\
(9.24)\end{array}$ & 0.982 & $\begin{array}{l}-0.003 \\
(-1.08)\end{array}$ & $\begin{array}{l}0.020 \\
(6.88)\end{array}$ & $\begin{array}{l}0.026 \\
(8.92)\end{array}$ & 0.981 & $\begin{array}{l}-0.028 \\
(-5.89)\end{array}$ & $\begin{array}{c}0.062 \\
(13.25)\end{array}$ & $\begin{array}{c}0.114 \\
(24.33)\end{array}$ & 0.915 \\
\hline 9 & $\begin{array}{l}-0.027 \\
(-4.70)\end{array}$ & $\begin{array}{l}0.045 \\
(7.66)\end{array}$ & $\begin{array}{c}0.062 \\
(10.46)\end{array}$ & 0.979 & $\begin{array}{l}-0.012 \\
(-4.15)\end{array}$ & $\begin{array}{l}0.023 \\
(7.93) \\
\end{array}$ & $\begin{array}{c}0.030 \\
(10.03) \\
\end{array}$ & 0.976 & $\begin{array}{l}-0.037 \\
(-7.88) \\
\end{array}$ & $\begin{array}{c}0.066 \\
(14.05) \\
\end{array}$ & $\begin{array}{c}0.124 \\
(26.41) \\
\end{array}$ & 0.900 \\
\hline 10 & $\begin{array}{l}-0.008 \\
(-1.36)\end{array}$ & $\begin{array}{l}0.043 \\
(7.27)\end{array}$ & $\begin{array}{c}0.066 \\
(11.24)\end{array}$ & 0.976 & $\begin{array}{l}-0.010 \\
(-3.54)\end{array}$ & $\begin{array}{l}0.024 \\
(8.10)\end{array}$ & $\begin{array}{c}0.031 \\
(10.56)\end{array}$ & 0.973 & $\begin{array}{l}-0.005 \\
(-1.05)\end{array}$ & $\begin{array}{c}0.049 \\
(10.51)\end{array}$ & $\begin{array}{c}0.085 \\
(18.12)\end{array}$ & 0.953 \\
\hline \multirow[t]{2}{*}{11} & $\begin{array}{l}-0.010 \\
(-1.62)\end{array}$ & $\begin{array}{l}0.046 \\
(7.80) \\
\end{array}$ & $\begin{array}{c}0.068 \\
(11.61)\end{array}$ & 0.974 & $\begin{array}{l}-0.001 \\
(-0.37)\end{array}$ & $\begin{array}{l}0.025 \\
(8.41) \\
\end{array}$ & $\begin{array}{c}0.036 \\
(12.04)\end{array}$ & 0.965 & $\begin{array}{l}-0.009 \\
(-1.88)\end{array}$ & $\begin{array}{c}0.048 \\
(10.11)\end{array}$ & $\begin{array}{c}0.083 \\
(17.70)\end{array}$ & 0.955 \\
\hline & \multicolumn{4}{|c|}{ Black alder } & \multicolumn{4}{|c|}{ White alder } & \multicolumn{4}{|c|}{ Aspen } \\
\hline 1 & $\begin{array}{l}0.003 \\
(0.71)\end{array}$ & $\begin{array}{l}0.023 \\
(5.50)\end{array}$ & $\begin{array}{l}0.032 \\
(7.40)\end{array}$ & 0.979 & $\begin{array}{l}-0.004 \\
(-1.98)\end{array}$ & $\begin{array}{l}0.022 \\
(9.79)\end{array}$ & $\begin{array}{c}0.032 \\
(14.63)\end{array}$ & 0.930 & $\begin{array}{l}-0.016 \\
(-2.66)\end{array}$ & $\begin{array}{c}0.041 \\
(6.89)\end{array}$ & $\begin{array}{l}0.057 \\
(9.51)\end{array}$ & 0.983 \\
\hline 2 & $\begin{array}{l}0.014 \\
(3.28)\end{array}$ & $\begin{array}{l}0.026 \\
(6.16) \\
\end{array}$ & $\begin{array}{l}0.032 \\
(7.43)\end{array}$ & 0.979 & $\begin{array}{l}-0.006 \\
(-2.91)\end{array}$ & $\begin{array}{c}0.024 \\
(10.72)\end{array}$ & $\begin{array}{c}0.035 \\
(15.60)\end{array}$ & 0.916 & $\begin{array}{l}-0.004 \\
(-0.75)\end{array}$ & $\begin{array}{l}0.040 \\
(6.67)\end{array}$ & $\begin{array}{l}0.058 \\
(9.68)\end{array}$ & 0.982 \\
\hline 3 & $\begin{array}{l}-0.001 \\
(-0.15)\end{array}$ & $\begin{array}{l}0.025 \\
(5.86) \\
\end{array}$ & $\begin{array}{l}0.033 \\
(7.78)\end{array}$ & 0.977 & $\begin{array}{l}-0.003 \\
(-1.33)\end{array}$ & $\begin{array}{c}0.023 \\
(10.45)\end{array}$ & $\begin{array}{c}0.033 \\
(15.12)\end{array}$ & 0.925 & $\begin{array}{l}-0.015 \\
(-2.50)\end{array}$ & $\begin{array}{l}0.043 \\
(7.18)\end{array}$ & $\begin{array}{l}0.059 \\
(9.96) \\
\end{array}$ & 0.981 \\
\hline 4 & $\begin{array}{l}0.006 \\
(1.52)\end{array}$ & $\begin{array}{l}0.025 \\
(5.97)\end{array}$ & $\begin{array}{l}0.034 \\
(7.90)\end{array}$ & 0.976 & $\begin{array}{l}0.007 \\
(3.13)\end{array}$ & $\begin{array}{l}0.020 \\
(9.25)\end{array}$ & $\begin{array}{c}0.029 \\
(13.27)\end{array}$ & 0.942 & $\begin{array}{l}-0.004 \\
(-0.69)\end{array}$ & $\begin{array}{c}0.042 \\
(7.09)\end{array}$ & $\begin{array}{c}0.062 \\
(10.48)\end{array}$ & 0.979 \\
\hline 5 & $\begin{array}{l}-0.001 \\
(-0.25)\end{array}$ & $\begin{array}{l}0.024 \\
(5.58)\end{array}$ & $\begin{array}{l}0.032 \\
(7.47)\end{array}$ & 0.979 & $\begin{array}{c}-0.007 \\
(3.18)\end{array}$ & $\begin{array}{c}0.023 \\
(10.47)\end{array}$ & $\begin{array}{c}0.035 \\
(15.70)\end{array}$ & 0.919 & $\begin{array}{l}-0.022 \\
(-3.73)\end{array}$ & $\begin{array}{l}0.042 \\
(7.10)\end{array}$ & $\begin{array}{l}0.056 \\
(9.40)\end{array}$ & 0.983 \\
\hline
\end{tabular}


Table 3. Cont.

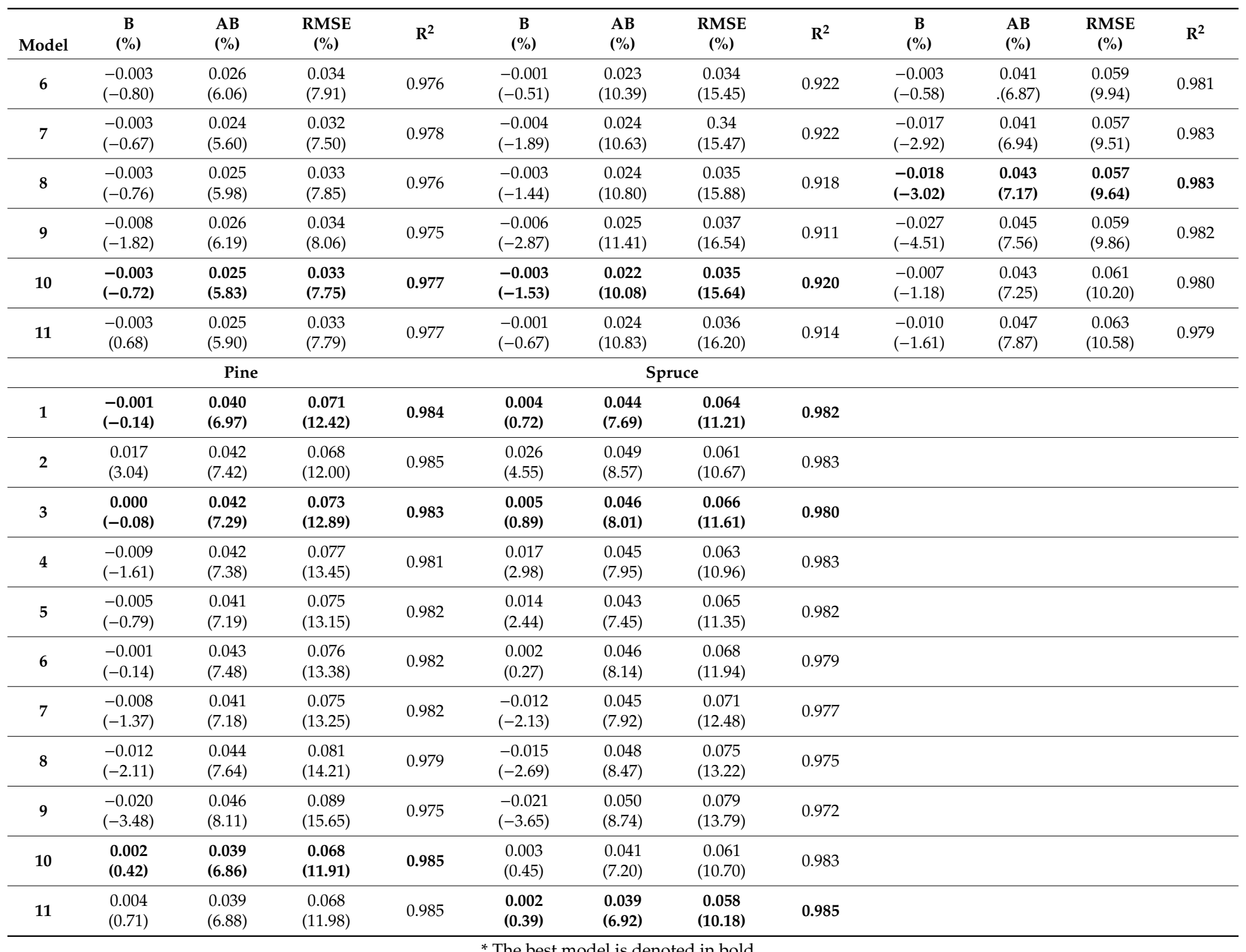

* The best model is denoted in bold. 
In Tables 2 and 3, when the validation dataset was used, the values of $R^{2}$ and the RMSE were obtained with all three modeling techniques (1. Models 1, 2, 4, 5, 7; 2. Models 3, 6, 8; 3. Models 9-11), show model fitness that is very similar for the both diameter and volume predictions. With the division of the models into three groups, overall, the values of the statistical measures were similar among the SDE models (M3, M6, and M8) and the regression models (M9 to M11), while the SDE models (M1, M2, M4, M5, M7) led to larger $\mathrm{R}^{2}$ and smaller RMSE values (Tables 2 and 3). With the division into three model groups, the optimal models, M1, M3, and M10 (optimal within each group), were selected based on all statistical measures, the visualization of residuals, and the model simplicity. The volume predictions by the stem taper models had very similar statistical measures (see Table 3). However, the diameter predictions by the SDE taper models that use the additional measurement of the fixed tree diameter at stem height $h=0$ were superior to the others.

\section{Discussion}

\subsection{Final Fitting}

Stem taper models fitted on a more complete dataset than the estimation dataset will naturally produce better estimates for all model parameters. In this study, any recruitment is no longer possible; thus, the next best solution is to extend the range of the estimation dataset by adding the validation dataset and renewing the parameter estimation procedures. Table 4 contains the estimated parameters of the best taper models, Models 1, 3 and 10, for each species group. All parameter estimates are significant $(p<0.05)$.

Statistical measures the goodness of fit for the diameter and volume using the stem diameter measurements from the complete dataset for all species group are presented in Tables 5 and 6, respectively. For all species groups, the best stem taper models, Models 1, 3 and 10, are not biased for the diameter predictions. For volume prediction with SDEs, Models 1 and 3 (spruce tree species group) and the regression model, Model 10 (oak tree species group), feature a slight bias. All tree species models produced percent root-mean-square error values of $6.71 \%$ to $10.75 \%$ and $7.91 \%$ to $18.02 \%$ for the diameter and volume predictions, respectively. SDE Model 1 produced superior statistical measures for all tree species groups. 
Table 4. Estimates of the fixed effect parameters for all fitted stem taper models.

\begin{tabular}{|c|c|c|c|c|c|c|c|c|c|c|c|}
\hline \multirow{2}{*}{ Species } & \multirow{2}{*}{ Model } & \multicolumn{9}{|c|}{ Parameters of Fitted Models } & \multirow[b]{2}{*}{$\mathbf{a}_{0}, \mathbf{a}_{1}$} \\
\hline & & $\alpha_{\mathrm{B}} \sim \beta_{1}{ }^{*}$ & $\beta_{B} \sim \beta_{2} *$ & $\sigma_{\mathbf{B}} \sim \beta_{3}{ }^{*}$ & $\alpha_{M} \sim \beta_{4} *$ & $\beta_{M} \sim \beta_{5} *$ & $\sigma_{M} \sim \beta_{6} *$ & $\alpha_{\mathrm{T}} \sim \beta_{7} *$ & $\beta_{\mathrm{T}} \sim \beta_{8} *$ & $\sigma_{\mathrm{T}} \sim \beta_{9} *$ & \\
\hline \multirow{3}{*}{ Oak } & 1 & 0.1813 & 77.788 & 0.1977 & -0.8322 & - & 0.1420 & 1.3975 & 1.3740 & 0.1661 & $1.3 / \mathrm{h} ; 0.48$ \\
\hline & 3 & 0.1750 & 78.035 & 0.2000 & -0.8459 & - & 0.1433 & 1.4723 & 1.2892 & 0.1696 & $1.3 / \mathrm{h} ; 0.53$ \\
\hline & 10 & 0.3396 & 0.9568 & 0.9927 & 0.3694 & 0.0122 & -0.974 & 0.1144 & -1.5853 & 9.0317 & 0.15 \\
\hline \multirow{3}{*}{ Ash } & 1 & -0.1913 & 54.172 & 0.2090 & -0.8921 & - & 0.1440 & 1.4213 & 1.2293 & 0.1757 & $1.3 / \mathrm{h} ; 0.44$ \\
\hline & 3 & -0.7740 & 41.592 & 0.1643 & -0.8887 & - & 0.1422 & 1.3468 & 1.3074 & 0.1818 & $1.3 / \mathrm{h} ; 0.47$ \\
\hline & 10 & 0.2545 & 0.6893 & 1.0029 & 0.4249 & -0.356 & -1.000 & 0.0043 & -0.1250 & 9.2630 & 0.13 \\
\hline \multirow{3}{*}{ Birch } & 1 & 0.1057 & 116.88 & 0.1122 & -1.1217 & - & 0.2426 & 0.5635 & 4.8869 & 0.2920 & $1.3 / \mathrm{h} ; 0.71$ \\
\hline & 3 & 0.1384 & 123.99 & 0.1161 & -1.1217 & - & 0.2426 & 0.5635 & 4.8869 & 0.2920 & $1.3 / \mathrm{h} ; 0.71$ \\
\hline & 10 & 0.3393 & 0.9509 & 0.9908 & 0.3502 & -0.018 & -0.9653 & 0.0869 & -1.3239 & 9.6494 & 0.13 \\
\hline \multirow{3}{*}{ Black alder } & 1 & -0.0274 & 51.275 & 0.2177 & -0.8009 & - & 0.1381 & 0.9267 & 2.7227 & 0.1784 & $1.3 / \mathrm{h} ; 0.52$ \\
\hline & 3 & 0.0406 & 53.403 & 0.2303 & -0.7958 & - & 0.1385 & 0.9362 & 2.6848 & 0.1768 & $1.3 / \mathrm{h} ; 0.51$ \\
\hline & 10 & -0.0023 & 0.9430 & 0.9688 & -0.2615 & 0.4868 & -1.434 & 0.1021 & -0.9516 & 6.9532 & 0.25 \\
\hline \multirow{3}{*}{ White alder } & 1 & -0.0910 & 46.120 & 0.2233 & -0.8821 & - & 0.1511 & 1.8751 & 0.9261 & 0.1402 & $1.3 / \mathrm{h} ; 0.58$ \\
\hline & 3 & -0.1245 & 45.548 & 0.2252 & -1.8821 & - & 0.1511 & 1.8751 & 0.9261 & 0.1402 & $1.3 / \mathrm{h} ; 0.57$ \\
\hline & 10 & 0.0586 & 1.1558 & 0.9636 & 0.3859 & 0.0784 & -0.9266 & 0.2623 & -2.9388 & 6.6790 & 0.25 \\
\hline \multirow{3}{*}{ Aspen } & 1 & 0.2800 & 84.246 & 0.2826 & -0.8373 & - & 0.1403 & 1.3975 & 1.3740 & 0.1661 & $1.3 / \mathrm{h} ; 0.48$ \\
\hline & 3 & 0.4664 & 98.204 & 0.3066 & -0.8373 & - & 0.1403 & 1.3975 & 1.3740 & 0.1661 & $1.3 / \mathrm{h} ; 0.48$ \\
\hline & 10 & 0.3286 & 0.9348 & 0.9907 & 0.4456 & 0.1650 & -1.1073 & 0.1462 & -1.5230 & 7.4188 & 0.26 \\
\hline \multirow{3}{*}{ Pine } & 1 & 0.2453 & 68.813 & 0.2748 & -1.0220 & - & 0.1752 & 0.7136 & 3.8005 & 0.2259 & $1.3 / \mathrm{h} ; 0.71$ \\
\hline & 3 & 0.6334 & 91.042 & 0.3203 & -1.0220 & - & 0.1752 & 0.7136 & 3.8005 & 0.2259 & $1.3 / \mathrm{h} ; 0.71$ \\
\hline & 10 & 0.0734 & 1.1571 & 0.9282 & -0.2450 & 0.5821 & -1.3718 & 0.3076 & -1.9119 & 5.0282 & 0.3 \\
\hline \multirow{3}{*}{ Spruce } & 1 & 0.2389 & 80.429 & 0.2498 & -0.8388 & - & 0.1377 & 0.7944 & 3.3469 & 0.2261 & $1.3 / \mathrm{h} ; 0.59$ \\
\hline & 3 & 0.2106 & 78.123 & 0.2481 & -0.8297 & - & 0.1360 & 0.8081 & 3.2693 & 0.2243 & $1.3 / \mathrm{h} ; 0.58$ \\
\hline & 10 & 0.3376 & 0.9468 & 0.9578 & -0.2098 & 0.1220 & -1.1860 & 0.0339 & -0.8099 & 7.9206 & 0.25 \\
\hline
\end{tabular}


Table 5. Statistical measures for all fitted stem diameter models.

\begin{tabular}{|c|c|c|c|c|c|c|c|c|c|c|c|c|}
\hline \multirow[t]{2}{*}{ Model } & $\begin{array}{c}\text { B } \\
(\%)\end{array}$ & $\begin{array}{l}A B \\
(\%)\end{array}$ & $\begin{array}{c}\text { RMSE } \\
(\%)\end{array}$ & $\mathbf{R}^{2}$ & $\begin{array}{c}\text { B } \\
(\%)\end{array}$ & $\begin{array}{l}A B \\
(\%)\end{array}$ & $\begin{array}{c}\text { RMSE } \\
(\%)\end{array}$ & $\mathbf{R}^{2}$ & $\begin{array}{c}\text { B } \\
(\%)\end{array}$ & $\begin{array}{l}A B \\
(\%)\end{array}$ & $\begin{array}{c}\text { RMSE } \\
(\%)\end{array}$ & $\mathbf{R}^{2}$ \\
\hline & \multicolumn{4}{|c|}{ Oak } & \multicolumn{4}{|c|}{ Ash } & \multicolumn{4}{|c|}{ Birch } \\
\hline 1 & $\begin{array}{l}-0.030 \\
(-0.19)\end{array}$ & $\begin{array}{l}0.800 \\
(5.13)\end{array}$ & $\begin{array}{l}1.275 \\
(8.18)\end{array}$ & 0.985 & $\begin{array}{l}-0.055 \\
(-0.43)\end{array}$ & $\begin{array}{l}0.641 \\
(5.00)\end{array}$ & $\begin{array}{l}0.966 \\
(7.53)\end{array}$ & 0.986 & $\begin{array}{l}-0.048 \\
(-0.30)\end{array}$ & $\begin{array}{l}1.021 \\
(6.29)\end{array}$ & $\begin{array}{c}1.641 \\
(10.11)\end{array}$ & 0.974 \\
\hline 3 & $\begin{array}{l}0.000 \\
(0.00)\end{array}$ & $\begin{array}{l}0.958 \\
(6.15)\end{array}$ & $\begin{array}{l}1.498 \\
(9.61)\end{array}$ & 0.979 & $\begin{array}{l}-0.040 \\
(-0.31)\end{array}$ & $\begin{array}{l}0.860 \\
(6.71)\end{array}$ & $\begin{array}{c}1.379 \\
(10.75)\end{array}$ & 0.971 & $\begin{array}{l}-0.053 \\
(-0.33)\end{array}$ & $\begin{array}{l}1.201 \\
(7.39)\end{array}$ & $\begin{array}{c}1.855 \\
(11.42)\end{array}$ & 0.967 \\
\hline \multirow[t]{2}{*}{10} & $\begin{array}{l}0.000 \\
(0.00)\end{array}$ & $\begin{array}{l}0.971 \\
(6.23)\end{array}$ & $\begin{array}{l}1.468 \\
(9.43)\end{array}$ & 0.980 & $\begin{array}{l}0.000 \\
(0.00)\end{array}$ & $\begin{array}{l}0.864 \\
(6.74)\end{array}$ & $\begin{array}{c}1.358 \\
(10.59)\end{array}$ & 0.972 & $\begin{array}{l}0.000 \\
(0.00)\end{array}$ & $\begin{array}{l}1.164 \\
(7.17)\end{array}$ & $\begin{array}{c}1.674 \\
(10.31)\end{array}$ & 0.973 \\
\hline & \multicolumn{4}{|c|}{ Black alder } & \multicolumn{4}{|c|}{ White alder } & \multicolumn{4}{|c|}{ Aspen } \\
\hline 1 & $\begin{array}{l}-0.005 \\
(-0.03)\end{array}$ & $\begin{array}{l}0.673 \\
(4.33)\end{array}$ & $\begin{array}{l}1.043 \\
(6.71)\end{array}$ & 0.985 & $\begin{array}{l}-0.007 \\
(-0.06)\end{array}$ & $\begin{array}{l}0.563 \\
(4.54)\end{array}$ & $\begin{array}{l}0.860 \\
(6.94)\end{array}$ & 0.988 & $\begin{array}{l}-0.023 \\
(-0.15)\end{array}$ & $\begin{array}{l}0.807 \\
(5.21)\end{array}$ & $\begin{array}{l}1.285 \\
(8.30)\end{array}$ & 0.985 \\
\hline 3 & $\begin{array}{l}0.003 \\
(0.02) \\
\end{array}$ & $\begin{array}{l}0.831 \\
(5.17) \\
\end{array}$ & $\begin{array}{l}1.271 \\
(7.91) \\
\end{array}$ & 0.979 & $\begin{array}{l}-0.006 \\
(-0.05) \\
\end{array}$ & $\begin{array}{l}0.719 \\
(5.80) \\
\end{array}$ & $\begin{array}{l}1.150 \\
(9.28)\end{array}$ & 0.978 & $\begin{array}{l}-0.014 \\
(-0.09)\end{array}$ & $\begin{array}{l}0.966 \\
(6.23) \\
\end{array}$ & $\begin{array}{l}1.508 \\
(9.74) \\
\end{array}$ & 0.979 \\
\hline \multirow[t]{2}{*}{10} & $\begin{array}{l}0.000 \\
(0.00)\end{array}$ & $\begin{array}{l}0.864 \\
(5.38)\end{array}$ & $\begin{array}{l}1.275 \\
(7.94)\end{array}$ & 0.979 & $\begin{array}{l}0.000 \\
(0.00)\end{array}$ & $\begin{array}{l}0.735 \\
(5.93)\end{array}$ & $\begin{array}{l}1.124 \\
(9.06)\end{array}$ & 0.979 & $\begin{array}{l}0.000 \\
(0.00)\end{array}$ & $\begin{array}{l}0.979 \\
(6.32)\end{array}$ & $\begin{array}{l}1.480 \\
(9.55)\end{array}$ & 0.980 \\
\hline & & Pine & & & & Sprt & & & & & & \\
\hline 1 & $\begin{array}{l}0.005 \\
(0.03)\end{array}$ & $\begin{array}{l}0.855 \\
(4.83)\end{array}$ & $\begin{array}{l}1.316 \\
(7.44)\end{array}$ & 0.986 & $\begin{array}{l}0.009 \\
(0.05)\end{array}$ & $\begin{array}{l}0.830 \\
(4.94)\end{array}$ & $\begin{array}{l}1.253 \\
(7.45)\end{array}$ & 0.986 & & & & \\
\hline 3 & $\begin{array}{l}0.002 \\
(0.01)\end{array}$ & $\begin{array}{l}0.991 \\
(5.60)\end{array}$ & $\begin{array}{l}1.495 \\
(8.46)\end{array}$ & 0.982 & $\begin{array}{l}0.026 \\
(0.16)\end{array}$ & $\begin{array}{l}1.011 \\
(6.01)\end{array}$ & $\begin{array}{l}1.578 \\
(9.38)\end{array}$ & 0.978 & & & & \\
\hline 10 & $\begin{array}{l}0.000 \\
(0.00)\end{array}$ & $\begin{array}{l}0.947 \\
(5.35)\end{array}$ & $\begin{array}{l}1.371 \\
(7.75)\end{array}$ & 0.985 & $\begin{array}{l}0.000 \\
(0.00)\end{array}$ & $\begin{array}{l}0.993 \\
(5.90)\end{array}$ & $\begin{array}{l}1.508 \\
(8.96)\end{array}$ & 0.980 & & & & \\
\hline
\end{tabular}


Table 6. Statistical measures for all fitted volume models.

\begin{tabular}{|c|c|c|c|c|c|c|c|c|c|c|c|c|}
\hline \multirow[t]{2}{*}{ Model } & $\begin{array}{c}\text { B } \\
(\%)\end{array}$ & $\begin{array}{l}\mathrm{AB} \\
(\%)\end{array}$ & $\begin{array}{c}\text { RMSE } \\
(\%)\end{array}$ & $\mathbf{R}^{2}$ & $\begin{array}{c}\text { B } \\
(\%)\end{array}$ & $\begin{array}{l}A B \\
(\%)\end{array}$ & $\begin{array}{c}\text { RMSE } \\
(\%)\end{array}$ & $\mathbf{R}^{2}$ & $\begin{array}{c}\text { B } \\
(\%)\end{array}$ & $\begin{array}{l}A B \\
(\%)\end{array}$ & $\begin{array}{c}\text { RMSE } \\
(\%)\end{array}$ & $\mathbf{R}^{2}$ \\
\hline & Oak & Ash & Birch & & & & & & & & & \\
\hline 1 & $\begin{array}{l}0.007 \\
(1.39)\end{array}$ & $\begin{array}{l}0.040 \\
(7.76)\end{array}$ & $\begin{array}{c}0.060 \\
(11.59)\end{array}$ & 0.983 & $\begin{array}{l}0.005 \\
(1.75)\end{array}$ & $\begin{array}{l}0.020 \\
(7.01)\end{array}$ & $\begin{array}{l}0.025 \\
(8.92)\end{array}$ & 0.981 & $\begin{array}{l}0.000 \\
(0.06)\end{array}$ & $\begin{array}{c}0.051 \\
(10.39)\end{array}$ & $\begin{array}{c}0.088 \\
(16.93)\end{array}$ & 0.958 \\
\hline 3 & $\begin{array}{l}0.009 \\
(1.74)\end{array}$ & $\begin{array}{l}0.041 \\
(7.81)\end{array}$ & $\begin{array}{c}0.060 \\
(11.57)\end{array}$ & 0.984 & $\begin{array}{l}0.004 \\
(1.46)\end{array}$ & $\begin{array}{l}0.020 \\
(7.24)\end{array}$ & $\begin{array}{l}0.026 \\
(9.19)\end{array}$ & 0.980 & $\begin{array}{l}0.000 \\
(0.06)\end{array}$ & $\begin{array}{c}0.052 \\
(10.48)\end{array}$ & $\begin{array}{c}0.089 \\
(18.02)\end{array}$ & 0.956 \\
\hline \multirow[t]{2}{*}{10} & $\begin{array}{l}0.011 \text { * } \\
(2.17)\end{array}$ & $\begin{array}{l}0.040 \\
(7.68)\end{array}$ & $\begin{array}{c}0.059 \\
(11.42)\end{array}$ & 0.984 & $\begin{array}{l}0.006 \\
(2.06)\end{array}$ & $\begin{array}{l}0.020 \\
(7.18)\end{array}$ & $\begin{array}{l}0.025 \\
(8.99)\end{array}$ & 0.981 & $\begin{array}{l}0.003 \\
(0.54)\end{array}$ & $\begin{array}{l}0.045 \\
(9.12)\end{array}$ & $\begin{array}{c}0.075 \\
(15.27)\end{array}$ & 0.969 \\
\hline & Black alder & White alder & Aspen & & & & & & & & & \\
\hline 1 & $\begin{array}{l}0.005 \\
(1.08)\end{array}$ & $\begin{array}{l}0.027 \\
(5.91)\end{array}$ & $\begin{array}{l}0.036 \\
(7.91)\end{array}$ & 0.982 & $\begin{array}{l}0.005 \\
(2.25)\end{array}$ & $\begin{array}{l}0.017 \\
(7.27)\end{array}$ & $\begin{array}{c}0.028 \\
(12.06)\end{array}$ & 0.967 & $\begin{array}{l}0.009 \\
(1.66)\end{array}$ & $\begin{array}{l}0.041 \\
(7.83)\end{array}$ & $\begin{array}{c}0.061 \\
(11.63)\end{array}$ & 0.983 \\
\hline 3 & $\begin{array}{l}0.004 \\
(0.96)\end{array}$ & $\begin{array}{l}0.027 \\
(6.01)\end{array}$ & $\begin{array}{l}0.037 \\
(8.04)\end{array}$ & 0.981 & $\begin{array}{l}0.005 \\
(2.15)\end{array}$ & $\begin{array}{l}0.017 \\
(7.25)\end{array}$ & $\begin{array}{c}0.028 \\
(11.87)\end{array}$ & 0.968 & $\begin{array}{l}0.009 \\
(1.77)\end{array}$ & $\begin{array}{l}0.041 \\
(7.85)\end{array}$ & $\begin{array}{c}0.061 \\
(11.61)\end{array}$ & 0.983 \\
\hline 10 & $\begin{array}{l}0.002 \\
(0.36)\end{array}$ & $\begin{array}{l}0.027 \\
(5.89)\end{array}$ & $\begin{array}{l}0.037 \\
(8.06)\end{array}$ & 0.981 & $\begin{array}{l}-0.003 \\
(-1.53)\end{array}$ & $\begin{array}{l}0.016 \\
(6.97)\end{array}$ & $\begin{array}{c}0.030 \\
(12.60)\end{array}$ & 0.964 & $\begin{array}{l}0.008 \\
(1.60)\end{array}$ & $\begin{array}{l}0.040 \\
(7.65)\end{array}$ & $\begin{array}{c}0.059 \\
(11.84)\end{array}$ & 0.984 \\
\hline Pine & Spruce & & & & & & & & & & & \\
\hline 1 & $\begin{array}{l}0.004 \\
(0.71)\end{array}$ & $\begin{array}{l}0.043 \\
(7.01)\end{array}$ & $\begin{array}{c}0.073 \\
(12.06)\end{array}$ & 0.983 & $\begin{array}{l}0.011^{*} \\
(1.95)\end{array}$ & $\begin{array}{l}0.044 \\
(7.64)\end{array}$ & $\begin{array}{c}0.065 \\
(11.48)\end{array}$ & 0.982 & & & & \\
\hline 3 & $\begin{array}{l}0.005 \\
(0.80)\end{array}$ & $\begin{array}{l}0.044 \\
(7.21)\end{array}$ & $\begin{array}{c}0.075 \\
(12.37)\end{array}$ & 0.982 & $\begin{array}{l}0.010 * \\
(1.74)\end{array}$ & $\begin{array}{l}0.044 \\
(7.26)\end{array}$ & $\begin{array}{c}0.067 \\
(11.80)\end{array}$ & 0.981 & & & & \\
\hline 10 & $\begin{array}{l}-0.007 \\
(-1.10)\end{array}$ & $\begin{array}{l}0.040 \\
(6.61)\end{array}$ & $\begin{array}{c}0.069 \\
(11.29)\end{array}$ & 0.985 & $\begin{array}{l}-0.001 \\
(-0.17)\end{array}$ & $\begin{array}{l}0.040 \\
(6.93)\end{array}$ & $\begin{array}{c}0.062 \\
(10.89)\end{array}$ & 0.983 & & & & \\
\hline
\end{tabular}




\subsection{Mean and Quantile Trajectories}

Foresters utilize information about the diameter at fixed distances from the base of the tree to predict the products of different diameter and length dimensions when the tree diameter at breast height and tree height measurements are known in advance. Stem taper equations can predict the diameter at any specified height, total stem volume, merchantable volume, and height at any specified stem diameter. In this section, the three best models for determining the tree diameter at any specified tree height are illustrated, showing SDE Models 1 and 3 and the regression generalized q-exponential equation in Model 10. The three finalized stem taper models are visualized in Figure 1. For the illustration plan, three stems from the complete dataset, corresponding to large, medium, and small trees were selected.
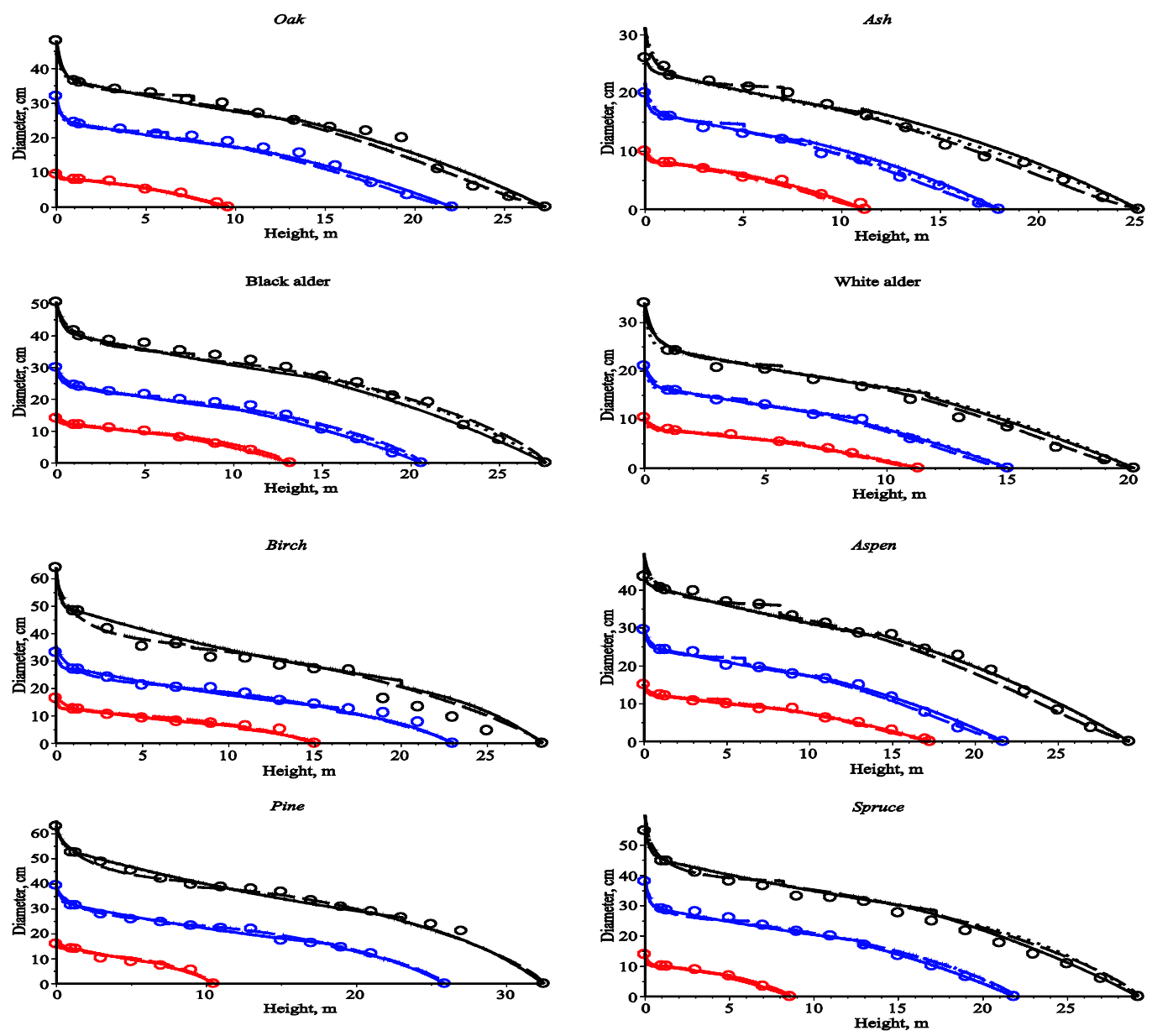

Figure 1. Comparison of fit observations for the evolution of diameter for the three stem taper models. Large tree is shown in black, medium tree in blue, and small tree in red. Model 1 is denoted by the solid line, Model 3 by the dotted line, and Model 10 by the dashed line.

Quantile regression is a method used to estimate the full conditional distribution of dependent variables [35,38]. Information about the distribution of diameters at any specified height is useful, for example, in understanding tree diameter dynamics against the tree height for the prediction of stem abnormality and for production management. The SDE stem taper models enables one to define any moment of stem diameter evolution. The main characteristics of the stem taper process focus on the 
mean function, defined by Equations (21)-(28), and the p-quantile $(0<p<1)$ functions, which, for Models 1 and 3, can be formulated in the following forms, respectively:

\section{Model 1}

$$
q u_{1}\left(x, d^{i}, h^{i}, p\right)=\left\{\begin{array}{l}
d^{i} \exp \left(\mu_{1}\left(\frac{x}{h^{i}} \mid \hat{\alpha}_{B}, \hat{\beta}_{B}, \hat{\sigma}_{B}, \frac{d_{0}^{i}}{d^{i}}\right)+\sqrt{v_{1}\left(\frac{x}{h^{i}} \mid \hat{\beta}_{B}, \hat{\sigma}_{B}\right)} \Phi_{p}^{-1}(0 ; 1)\right), 0 \leq \frac{x}{h^{i}}<\frac{1.3}{h^{i}} \\
d^{i} \exp \left(\mu_{2}\left(\frac{x}{h^{i}} \mid \hat{\alpha}_{M}, \hat{\sigma}_{M}, 1 .\right)+\sqrt{v_{2}\left(\frac{x}{h^{i}} \mid \hat{\sigma}_{M}\right)} \Phi_{p}^{-1}(0 ; 1)\right), \frac{1.3}{h^{i}} \leq \frac{x}{h^{i}}<a_{1} \\
d^{i} \Phi_{p}^{-1}\left(\mu_{3}\left(\frac{x}{h^{i}} \mid \hat{\alpha}_{T}, \hat{\beta}_{T}, 0\right) ; v_{3}\left(\frac{x}{h^{i}} \mid \hat{\beta}_{T}, \hat{\sigma}_{T}, 0\right)\right), a_{1} \leq \frac{x}{h^{i}} \leq 1
\end{array},\right.
$$

Model 3

$$
q u_{1}\left(x, d^{i}, h^{i}, p\right)=\left\{\begin{array}{l}
d^{i} \exp \left(L \Phi_{p}^{-1}\left(\hat{\mu}_{0} ; \hat{\sigma}_{0}^{2}\right)\left(\mu_{1}\left(\frac{x}{h^{i}} \mid \hat{\alpha}_{B}, \hat{\beta}_{B}, \hat{\sigma}_{B}, Q_{p}\right)+\sqrt{v_{1}\left(\frac{x}{h^{i}} \mid \hat{\beta}_{B}, \hat{\sigma}_{B}\right)} \Phi_{p}^{-1}(0 ; 1)\right)\right), \frac{x}{h^{i}}<\frac{1.3}{h^{i}} \\
d^{i} \exp \left(\mu_{2}\left(\frac{x}{h^{i}} \mid \hat{\alpha}_{M}, \hat{\sigma}_{M}, 1\right)+\sqrt{v_{2}\left(\frac{x}{h^{i}} \mid \hat{\sigma}_{M}\right)} \Phi_{p}^{-1}(0 ; 1)\right), \frac{1.3}{h^{i}} \leq \frac{x}{h^{i}}<a_{1} \\
d^{i} \Phi_{p}^{-1}\left(\mu_{3}\left(\frac{x}{h^{i}} \mid \hat{\alpha}_{T}, \hat{\beta}_{T}, 0\right) ; v_{3}\left(\frac{x}{h^{i}} \mid \hat{\beta}_{T}, \hat{\sigma}_{T}, 0\right)\right), a_{1} \leq \frac{x}{h^{i}} \leq 1
\end{array} .\right.
$$

Figure 2 shows the mean stem taper dynamic, as well as its 0.025 and 0.975 quantile dynamics, for three randomly selected stems from the complete dataset.
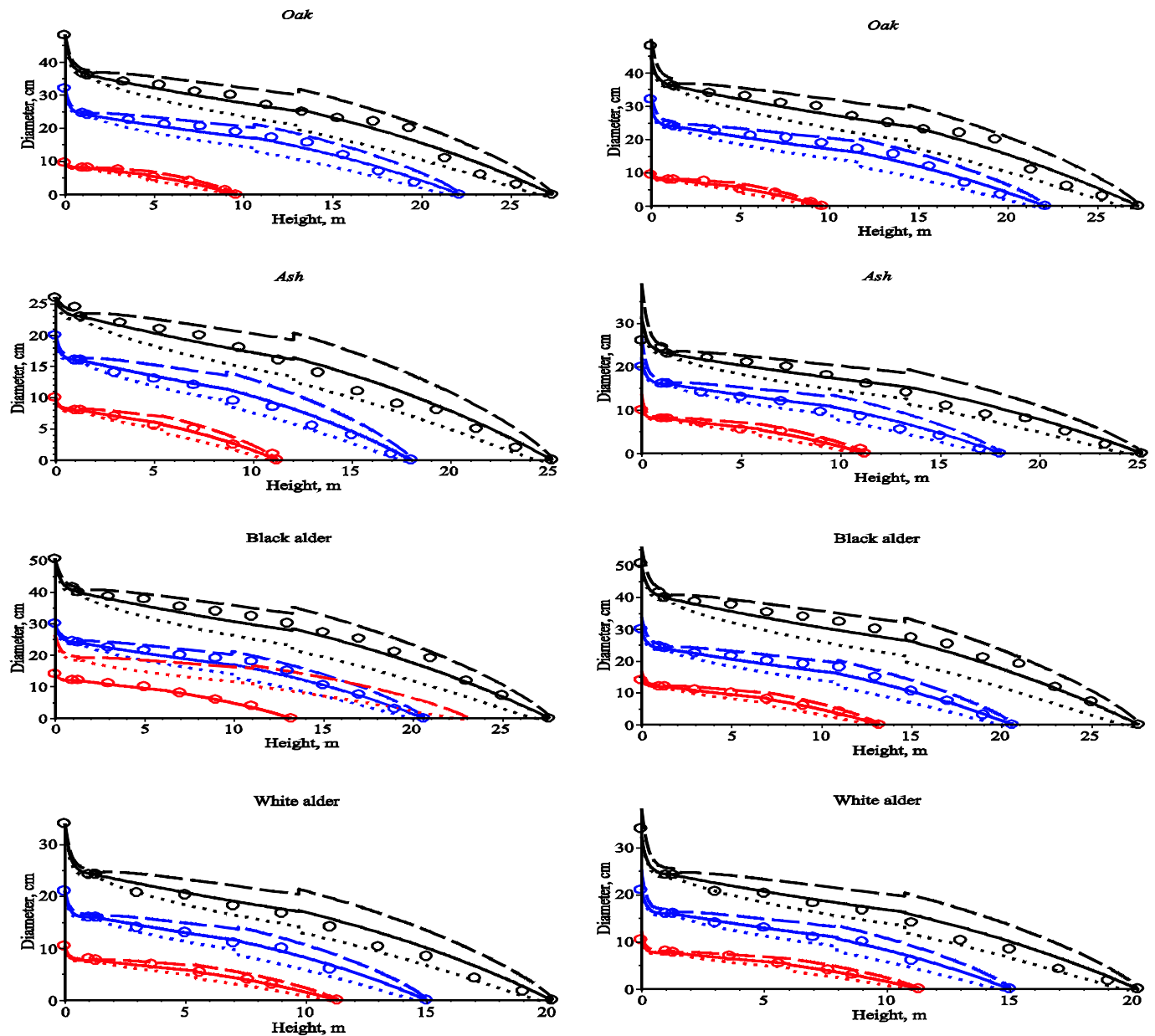

Figure 2. Cont. 

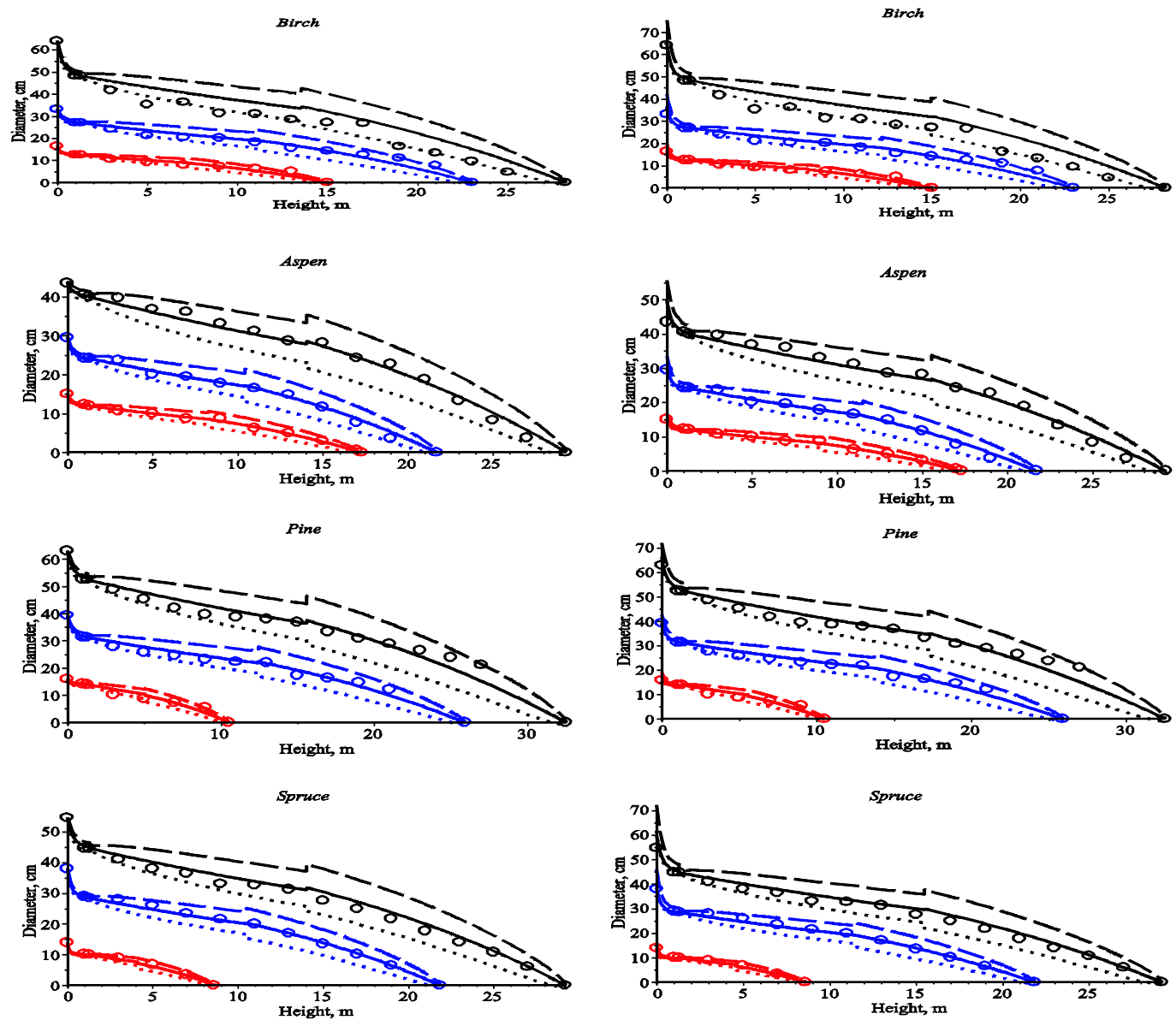

Figure 2. Evolution of the mean and $p$-quantile for stem diameter. Left shows Model 1, right shows Model 3. The large tree is denoted in black, the medium tree in blue, and the small tree in red. The mean trajectory is denoted with the solid line, the 0.025 -quantile trajectory is denoted with the dotted line, and the 0.975-quantile trajectory is denoted with the dashed line.

\section{Conclusions}

This study was conducted to develop a stem taper models of the eight dominant tree species in Lithuania (pine, spruce, oak, ash, birch, black alder, white alder, and aspen), by using SDEs. Classic stem taper equations are generally described as segmented polynomial and variable exponential models, which can be studied as a part of more sophisticated models. This work considers eight SDEs models that can be applied to practical situations. Two strategies have been proposed for managing the original SDE and producing eight different stochastic models. One of them is the symmetric Vasicek type stochastic process while the others are the asymmetric Gompertz and gamma type stochastic processes. The form of the SDE taper models was also designed to be consistent with common forest inventory practices, to give them practical as well as scientific value for tree profile evaluation. Quantiles equations of SDEs stem taper models, defined by Equations (46) and (47), enable us to some degree censor a sample population of trees by excluding trees which diverge from some idealized stem form. The proposed eight SDE stem taper models were compared to three regression stem taper equations based on data from the eight dominant tree species in Lithuania (pine, spruce, oak, ash, birch, black alder, white alder, and aspen). The results of this study indicated that the SDE stem taper Models 1 and 3 can be used to accurately predict the tree diameter at a specified height of the eight dominant tree species in Lithuania. 
Author Contributions: Conceptualization, P.R. and E.P.; methodology, E.P.; software, P.R.; validation, P.R., E.P. and M.N.; formal analysis, M.N. and M.A.; data curation, M.N., M.A., L.B., B.Š. and E.P; writing-original draft preparation, P.R., E.P. and M.N.; writing—review and editing, P.R., E.P., M.N., M.A., L.B. and B.Š. All authors have read and agreed to the published version of the manuscript.

Funding: This research received no external funding.

Acknowledgments: The authors are very grateful to the Lithuanian Association of Impartial Timber Scalers for the sample data set.

Conflicts of Interest: The authors declare no conflict of interest.

\section{References}

1. Bachelier, L. Théorie de la spéculation. Annales Scientifiques de l'École Normale Supérieure 1900, 3, 21-86. [CrossRef]

2. Nafidi, A.; Bahij, M.; Gutiérrez-Sánchez, R.; Achchab, B. Two-Parameter Stochastic Weibull Diffusion Model: Statistical Inference and Application to Real Modeling Example. Mathematics 2020, 8, 160. [CrossRef]

3. Rupšys, P.; Petrauskas, E. A New Paradigm in Modelling the Evolution of a Stand via the Distribution of Tree Sizes. Sci. Rep. 2017, 7, 15875. [CrossRef] [PubMed]

4. Visalga, G.; Rupšys, P.; Petrauskas, E. Influence of Noise on Decay Predictions in Standing Trees. AIP Conf. Proc. 2017, 1895, 030006.

5. Dürr, R.; Waldherr, S. A Novel Framework for Parameter and State Estimation of Multicellular Systems Using Gaussian Mixture Approximations. Processes 2018, 6, 187. [CrossRef]

6. Calatayud Gregori, J.; Chen-Charpentier, B.M.; Cortés López, J.C.; Jornet Sanz, M. Combining Polynomial Chaos Expansions and the Random Variable Transformation Technique to Approximate the Density Function of Stochastic Problems, Including Some Epidemiological Models. Symmetry 2019, 11, 43. [CrossRef]

7. Román-Román, P.; Romero, D.; Torres-Ruiz, F. A Diffusion Process to Model Generalized von Bertalanffy Growth Patterns: Fitting to Real Data. J. Theor. Biol. 2010, 263, 59-69. [CrossRef]

8. Cresson, J.; Sonner, S. A Note on a Derivation Method for SDE Models: Applications in Biology and Viability Criteria. Stoch. Anal. Appl. 2018, 36, 224-239. [CrossRef]

9. Ruse, M.G.; Samson, A.; Ditlevsen, S. Inference for biomedical data by using diffusion models with covariates and mixed effects. J. R. Stat. Soc. C Appl. 2020, 69, 167-193. [CrossRef]

10. Rupšys, P. The Use of Copulas to Practical Estimation of Multivariate Stochastic Differential Equation Mixed Effects Models. AIP Conf. Proc. 2015, 1684, 080011.

11. Rupšys, P.; Petrauskas, E. A Linkage among Tree Diameter, Height, Crown Base Height, and Crown Width 4-variate Distribution and Their Growth Models: A 4-variate Diffusion Process Approach. Forests 2017, 8 , 479. [CrossRef]

12. Rupšys, P. Modeling Dynamics of Structural Components of Forest Stands Based on Trivariate Stochastic Differential Equation. Forests 2019, 10, 506. [CrossRef]

13. Rupšys, P. Understanding the Evolution of Tree Size Diversity within the Multivariate Nonsymmetrical Diffusion Process and Information Measures. Mathematics 2019, 7, 761. [CrossRef]

14. Max, T.A.; Burkhart, H.E. Segmented Polynomial Regression Applied to Taper Models. For. Sci. 1976, 22, 283-289.

15. Kozak, A. My Last Words on Taper Equations. For. Chron. 2004, 80, 507-515. [CrossRef]

16. Petrauskas, E.; Bartkevičius, E.; Rupšys, P.; Memgaudas, R. The use of stochastic differential equations to describe stem taper and volume. Baltic For. 2013, 19, 43-151.

17. Bronisz, K.; Zasada, M. Comparison of Fixed- and Mixed-Effects Approaches to Taper Modeling for Scots Pine in West Poland. Forests 2019, 10, 975. [CrossRef]

18. Socha, J.; Netzel, P.; Cywicka, D. Stem Taper Approximation by Artificial Neural Network and a Regression Set Models. Forests 2020, 11, 79. [CrossRef]

19. Garcia, O. Dynamic Modelling of Tree Form. Math. Comput. For. Nat. Res. Sci. 2015, 7, 9-15.

20. Sone, K.; Suzuki, A.; Miyazawa, S.-I.; Noguchi, K.; Terashima, I. Maintenance mechanisms of the pipe model relationship and Leonardo da Vinci's rule in the branching architecture of Acer runerve trees. J. Plant Res. 2009, 122, 41-52. [CrossRef] 
21. Narmontas, M.; Rupšys, P.; Petrauskas, E. Models for Tree Taper Form: The Gompertz and Vasicek Diffusion Processes Framework. Symmetry 2020, 12, 80. [CrossRef]

22. Muhairwe, C.K. Tree form and taper variation over time for interior lodgepole pine. Can. J. For. Res. 1994, 24, 1904-1913. [CrossRef]

23. Sharma, M.; Burkhart, H.E. Selecting a Level of Conditioning for the Segmented Polynomial Taper Equation. For. Sci. 2002, 49, 324-330.

24. Kozak, A. A Variable-Exponent Taper Equation. Can. J. For. Res. 1988, 18, 1363-1368. [CrossRef]

25. Chiba, Y. A Quantitative Analysis of Stem Form and Crown Structure: The S-curve and its Application. Tree Phys. 1990, 7, 169-182. [CrossRef] [PubMed]

26. Ko, C.; Kang, J.T.; Son, Y.M.; Kim, D.-G. Estimating stem volume using stem taper equation for Quercus mongolica in South Korea. Forest Sci. Technol. 2019, 15, 58-62. [CrossRef]

27. Özçelik, R.; Brooks, J.R.; Jiang, L. Modeling stem profile of Lebanon cedar, Brutian pine, and Cilicica fir in Southern Turkey using nonlinear mixed-effects models. Eur. J. For. Res. 2011, 130, 613-621. [CrossRef]

28. Trincado, G.; Burkhart, H.E. A generalized approach for modeling and localizing stem profile curves. For. Sci. 2006, 52, 670-682.

29. Rupšys, P. Univariate and Bivariate Diffusion Models: Computational Aspects and Applications to Forestry. In Stochastic Differential Equations: Basics and Applications; Deangelo, T.G., Ed.; Nova Science Publisher's: New York, NY, USA, 2018; pp. 1-77.

30. Li, C. Maximum-Likelihood Estimation for Diffusion Processes via Closed-Form density Expansions. Ann. Statist. 2013, 41, 1350-1380. [CrossRef]

31. García, O. Estimating Reducible Stochastic Differential Equations by Conversion to a Least-Squares Problem. Comput. Stat. 2019, 34, 23-46. [CrossRef]

32. Picchini, U.; Gaetano, A.; Ditlevsen, S. Stochastic Differential Mixed-Effects Models. Scand. J. Stat. 2010, 37, 67-90. [CrossRef]

33. Jiang, L.; Liu, R. Segmented Taper Equations with Crown Ratio and Stand Density for Dahurian Larch (Larix gmelinii) in Northeastern China. J. For. Res. 2011, 22, 347. [CrossRef]

34. Dean, T.J. Neighbor and Height Effects on Crown Properties Associated with the Uniform-Stress Principle of Stem Formation. Forests 2018, 9, 334. [CrossRef]

35. Zhang, B.; Sajjad, S.; Chen, K.; Zhou, L.; Zhang, Y.; Yong, K.K.; Sun, Y. Predicting Tree Height-Diameter Relationship from Relative Competition Levels Using Quantile Regression Models for Chinese Fir (Cunninghamia lanceolata) in Fujian Province, China. Forests 2020, 11, 183. [CrossRef]

36. Monagan, M.B.; Geddes, K.O.; Heal, K.M.; Labahn, G.; Vorkoetter, S.M.; McCarron, J.; DeMarco, P. Maple Advanced Programming Guide; Maplesoft: Ontario, ON, Canada, 2007; p. 388.

37. Rupšys, P. Modeling Perspectives of Forest Growth and Yield: Framework of Multivariate Diffusion Process. AIP Conf. Proc. 2019, 2164, 060017.

38. Zarnani, A.; Karimi, S.; Musilek, P. Quantile Regression and Clustering Models of Prediction Intervals for Weather Forecasts: A Comparative Study. Forecasting 2019, 1, 169-188. [CrossRef]

(C) 2020 by the authors. Licensee MDPI, Basel, Switzerland. This article is an open access article distributed under the terms and conditions of the Creative Commons Attribution (CC BY) license (http://creativecommons.org/licenses/by/4.0/). 